\title{
Mild and General Conditions for the Cross-Coupling of Aryl Halides with Pentafluorobenzene and other Perfluoroaromatics
}

Marc Lafrance, Daniel Shore \& Keith Fagnou *

Center for Catalysis Research and Innovation, University of Ottawa, Department of Chemistry, 10 Marie Curie, Ottawa, Ontario, Canada K1N 6N5

Keith.Fagnou@science.uottawa.ca

\section{Supporting Information}

\section{General Methods:}

All experiments were carried out under an atmosphere of argon. ${ }^{1} \mathrm{H},{ }^{13} \mathrm{C}$ and ${ }^{19} \mathrm{~F}$ NMR were recorded in $\mathrm{CDCl}_{3}$ solutions using a Bruker AVANCE 400 spectrometer. Trifluorotoluene $(\delta=-67.73 \mathrm{ppm})$ was employed as an external standard in ${ }^{19} \mathrm{~F}$ NMR spectra ( $\mathrm{T} 1=20$ seconds). High-resolution mass spectra were obtained on a Kratos Concept IIH. Infra-Red analysis was performed with a Bruker EQUINOX 55. HPLC Grade pentane, $\mathrm{Et}_{2} \mathrm{O}$, hexane, and $\mathrm{CHCl}_{3}$ were employed. Isopropyl acetate was degassed with Argon before every use. Phosphonium salts were purchased from Strem, stored in a dessicator and used without further purification. Palladium sources were stored in a dessicator and were weighed out to air unless otherwise specified. Compounds $3^{1}, 4^{2}, 6^{2}, 10^{3}$, $16^{1}, 17^{1}, 18^{1}, 19^{4}, 20^{1}, 23^{5}, 25^{6}, 27^{3}, 28^{3}, 29^{7}$ exhibited identical spectral data to that reported, all other reagents and solvents were used as is from commercial sources.

\section{Table of Contents}

General Procedure S2

Characterization for Perfluorobiaryls S2-S12

Attached ${ }^{1} \mathrm{H} \&{ }^{13} \mathrm{C}$ Spectra 


\section{Products}

\section{General Procedure:}

$\mathrm{K}_{2} \mathrm{CO}_{3}$ (2.0 equiv.), 2-cyclohexylphosphino-2',6'-dimethoxy-1,1'-biphenyl (0.10 equiv.), $\mathrm{Pd}(\mathrm{OAc})_{2}$ (0.05 equiv.) were weighed to air and placed in a screw capped vial $(2 \mathrm{~mL})$ with a magnetic stir bar. The reaction vessel was evacuated and backfilled with argon (x3). The fluoroarene (1.5 eq.) and isopropylacetate (1M) were then added via syringe and the reaction was stirred for one minute. The aryl halide ( 1 equiv.) was then added and the reaction was heated to $80^{\circ} \mathrm{C}$ for 12 hours (If the aryl halide is a solid, it is added as a solution in $i$-PrOAc). Upon completion, the reaction was then cooled to room temperature and purified by loading the crude reaction mixture directly onto a short silica gel packed flash chromatography column typically using hexane or pentane/ether mixtures as the eluant.

\section{3'-ethyl-2,3,4,5,6-pentafluoro-1,1'-biphenyl (5)}<smiles>CCc1ccccc1-c1c(F)c(F)c(F)c(F)c1F</smiles>

The compound was prepared following the general procedure in $82 \%$ yield: $R_{f}=$ $0.46\left(\mathrm{SiO}_{2}, 100 \%\right.$ Hexane $)$; IR $\left(v_{\max } / \mathrm{cm}^{-1}\right): 2973,1522,1494,1062,989 ;{ }^{1} \mathrm{H}$ NMR $\left(400 \mathrm{MHz}, \mathrm{CDCl}_{3}, 293 \mathrm{~K}, \mathrm{TMS}\right): 1.10(3 \mathrm{H}, \mathrm{t}, J=7.6 \mathrm{~Hz}), 2.45(2 \mathrm{H}, \mathrm{q}, J=$ $7.6 \mathrm{~Hz}), 7.15(1 \mathrm{H}, \mathrm{dd}, J=7.5,1.0 \mathrm{~Hz}), 7.30(1 \mathrm{H}, \mathrm{ddd}, J=7.5,7.5,1.3 \mathrm{~Hz}), 7.40$ $(1 \mathrm{H}, \mathrm{dd}, J=7.5,1.1 \mathrm{~Hz}), 7.42(1 \mathrm{H}, \mathrm{ddd}, J=7.4,7.4,1.3 \mathrm{~Hz}) ;{ }^{13} \mathrm{C} N M R(100 \mathrm{MHz}$, $\left.\mathrm{CDCl}_{3}, 293 \mathrm{~K}, \mathrm{TMS}\right): 15.0,26.6,115.7\left(\mathrm{td}, J_{F}=20.3,4.0 \mathrm{~Hz}\right), 125.4,125.4,126.3$, 129.0, 130.0, 130.9, $137.8\left(\mathrm{dm}, J_{F}=253.2 \mathrm{~Hz}\right), 140.8\left(\mathrm{dm}, J_{F}=253.4 \mathrm{~Hz}\right), 143.6$, $144.4\left(\mathrm{dm}, J_{F}=245.8 \mathrm{~Hz}\right) ;{ }^{19} \mathrm{~F} \mathrm{NMR}\left(377 \mathrm{MHz}, \mathrm{CDCl}_{3}, 293 \mathrm{~K}, \mathrm{TMS}\right):-145.2(2 \mathrm{~F}$, $\left.\mathrm{dd}, J_{F}=23.2,8.2 \mathrm{~Hz}\right),-160.4\left(1 \mathrm{~F}, \mathrm{t}, J_{F}=21.3 \mathrm{~Hz}\right),-167.5\left(2 \mathrm{~F}, \mathrm{ddd}, J_{F}=23.1,21.6\right.$, 8.3Hz); HRMS calcd for $\mathrm{C}_{14} \mathrm{H}_{9} \mathrm{~F}_{5}(\mathrm{M}+)$ 272.0624; found: 272.0628 . 


\section{2'-methyl-4'fluoro-2,3,4,5,6-pentafluoro-1,1'-biphenyl (7)}<smiles>Cc1cc(F)ccc1-c1c(F)c(F)c(F)c(F)c1F</smiles>

The compound was prepared following the general procedure in $96 \%$ yield: $R_{f}=$ $0.46\left(\mathrm{SiO}_{2}, 100 \%\right.$ Hexane); IR $\left(v_{\max } / \mathrm{cm}^{-1}\right): 2931,1521,1493,1057,835 ;{ }^{1} \mathrm{H}$ NMR $\left(400 \mathrm{MHz}, \mathrm{CDCl}_{3}, 293 \mathrm{~K}, \mathrm{TMS}\right): 2.18,(3 \mathrm{H}, \mathrm{s}), 7.0(1 \mathrm{H}, \mathrm{ddd}, \mathrm{J}=8.3,8.3$, $2.7 \mathrm{~Hz}), 7.07(1 \mathrm{H}, \mathrm{dd}, J=9.5,2.5 \mathrm{~Hz}), 7.16(1 \mathrm{H}, \mathrm{dd}, J=8.5,5.7 \mathrm{~Hz}) ;{ }^{13} \mathrm{C} N M R$ $\left(100 \mathrm{MHz}, \mathrm{CDCl}_{3}, 293 \mathrm{~K}, \mathrm{TMS}\right): 19.8\left(\mathrm{~d}, J_{F}=1.1 \mathrm{~Hz}\right), 113.3\left(\mathrm{~d}, J_{F}=21.6 \mathrm{~Hz}\right)$, 114.4-114.8 (m), $117.5\left(\mathrm{~d}, J_{F}=21.6 \mathrm{~Hz}\right), 121.8\left(\mathrm{~d}, J_{F}=1.5 \mathrm{~Hz}\right), 132.4,\left(\mathrm{~d}, J_{F}=\right.$ 8.8Hz), 137.8, $\left(\mathrm{dm}, J_{F}=253.5 \mathrm{~Hz}\right), 140.9\left(\mathrm{dm}, J_{F}=253.8 \mathrm{~Hz}\right), 140.2\left(\mathrm{~d}, J_{F}=\right.$ 8.4Hz), $144.4\left(\mathrm{dm}, J_{F}=249.0 \mathrm{~Hz}\right), 150.5,150.6 ;{ }^{19} \mathrm{~F} \mathrm{NMR}\left(377 \mathrm{MHz}, \mathrm{CDCl}_{3}, 293 \mathrm{~K}\right.$, TMS): $-117.3(1 \mathrm{~F}, \mathrm{~s}),-145.4\left(2 \mathrm{~F}, \mathrm{dd}, J_{F}=23.4,8.7 \mathrm{~Hz}\right),-159.8\left(1 \mathrm{~F}, \mathrm{t}, J_{F}=\right.$ $20.7 \mathrm{~Hz}),-166.9\left(2 \mathrm{~F}, \mathrm{ddd}, \mathrm{J}_{F}=22.9,21.4,8.6 \mathrm{~Hz}\right) ; \mathrm{HRMS}$ calcd for $\mathrm{C}_{13} \mathrm{H}_{6} \mathrm{~F}_{6}(\mathrm{M}+)$ 276.0374; found: 276.0383 .

\section{2-methyl-1-(perfluorophenyl)naphthalene (8)}<smiles>Cc1ccc2ccccc2c1-c1c(F)c(F)c(F)c(F)c1F</smiles>

The compound was prepared following the general procedure in $96 \%$ yield: $\mathrm{mp}$ 82-85 ${ }^{\circ} \mathrm{C}\left(\mathrm{CHCl}_{3}\right) ; \mathrm{R}_{f}=0.34\left(\mathrm{SiO}_{2}, 100 \%\right.$ Hexane $) ; \mathrm{IR}\left(v_{\max } / \mathrm{cm}^{-1}\right): 3057,1521$, 1494, 1108, 987; ${ }^{1} \mathrm{H}$ NMR (400MHz, $\left.\mathrm{CDCl}_{3}, 293 \mathrm{~K}, \mathrm{TMS}\right): 2.28,(3 \mathrm{H}, \mathrm{s}), 7.29(1 \mathrm{H}$, $\mathrm{d}, J=7.8 \mathrm{~Hz}), 7.40-7.47(3 \mathrm{H}, \mathrm{m}), 7.85-7.89(2 \mathrm{H}, \mathrm{m}) ;{ }^{13} \mathrm{C} \mathrm{NMR}\left(100 \mathrm{MHz}, \mathrm{CDCl}_{3}\right.$, 293K, TMS): 20.4, $113.3\left(\mathrm{td}, J_{F}=20.6,3.9 \mathrm{~Hz}\right), 121.9\left(\mathrm{~d}, J_{F}=1.2 \mathrm{~Hz}\right), 124.1$, $125.5,127.1,128.4,128.4,129.8,132.1,132.2,136.0,137.9\left(\mathrm{dm}, J_{F}=253.9 \mathrm{~Hz}\right)$, 141.0, $\left(\mathrm{dm}, J_{F}=253.9 \mathrm{~Hz}\right), 144.4\left(\mathrm{dm}, J_{F}=246.8 \mathrm{~Hz}\right) ;{ }^{19} \mathrm{~F} \mathrm{NMR}\left(377 \mathrm{MHz}, \mathrm{CDCl}_{3}\right.$, 293K, TMS): -144.0 (2F, dd, $\left.J_{F}=23.6,9.1 \mathrm{~Hz}\right),-159.4\left(1 \mathrm{~F}, \mathrm{t}, J_{F}=21.2 \mathrm{~Hz}\right),-166.6$ 
(2F, ddd, $\left.J_{F}=24.2,21.9,9.3 \mathrm{~Hz}\right)$; HRMS calcd for $\mathrm{C}_{17} \mathrm{H}_{9} \mathrm{~F}_{5}(\mathrm{M}+$ ) 308.0624; found: 308.0607.

\section{2-(perfluorophenyl)biphenyl (9)}<smiles>Fc1c(F)c(F)c(-c2ccccc2-c2ccccc2)c(F)c1F</smiles>

The compound was prepared following the general procedure in $92 \%$ yield: $\mathrm{mp}$ $89-91^{\circ} \mathrm{C}\left(\mathrm{CHCl}_{3}\right) ; \mathrm{R}_{f}=0.33\left(\mathrm{SiO}_{2}, 100 \%\right.$ Hexane $) ; \mathrm{IR}\left(v_{\max } / \mathrm{cm}^{-1}\right): 3068,1522$, 1495, 988; ${ }^{1} \mathrm{H}$ NMR (400MHz, $\left.\mathrm{CDCl}_{3}, 293 \mathrm{~K}, \mathrm{TMS}\right):$ 7.12-7.14 (2H, m), 7.24-7.28 $(3 \mathrm{H}, \mathrm{m}), 7.32-7.34(1 \mathrm{H}, \mathrm{m}), 7.44-7.50(2 \mathrm{H}, \mathrm{m}), 7.53-7.57(1 \mathrm{H}, \mathrm{m}) ;{ }^{13} \mathrm{C} N M R$ (100MHz, $\left.\mathrm{CDCl}_{3}, 293 \mathrm{~K}, \mathrm{TMS}\right): 116.0$ (td, $\left.J_{F}=19.4,4.0 \mathrm{~Hz}\right), 125.0,127.5,127.5$, 128.2, 128.5, 129.8, 130.4, 131.1, $137.4\left(\mathrm{dm}, J_{F}=252.9 \mathrm{~Hz}\right), 140.4,140.6\left(\mathrm{dm}, J_{F}\right.$ $=253.4 \mathrm{~Hz}), 143.1,144.1\left(\mathrm{dm}, J_{F}=246.5 \mathrm{~Hz}\right) ;{ }^{19} \mathrm{~F} \mathrm{NMR}\left(377 \mathrm{MHz}, \mathrm{CDCl}_{3}, 293 \mathrm{~K}\right.$, TMS): $-145.1\left(2 \mathrm{~F}, \mathrm{dd}, J_{F}=24.5,9.5 \mathrm{~Hz}\right),-160.4\left(1 \mathrm{~F}, \mathrm{t}, J_{F}=20.8 \mathrm{~Hz}\right),-167.5(2 \mathrm{~F}$, ddd, $J_{F}=23.3,20.6,8.5 \mathrm{~Hz}$; HRMS calcd for $\mathrm{C}_{18} \mathrm{H}_{9} \mathrm{~F}_{5}(\mathrm{M}+)$ 320.0624; found: 320.0613 .

\section{1-(perfluorophenyl)naphthalene (11)}<smiles>Fc1c(F)c(F)c(-c2cccc3ccccc23)c(F)c1F</smiles>

The compound was prepared following the general procedure in $99 \%$ yield: $\mathrm{mp}$ 92-93 ${ }^{\circ} \mathrm{C}\left(\mathrm{CHCl}_{3}\right) ; \mathrm{R}_{f}=0.34\left(\mathrm{SiO}_{2}, 100 \%\right.$ Hexane $) ; \mathrm{IR}\left(v_{\max } / \mathrm{cm}^{-1}\right): 3066,1516$, 1493, 992; ${ }^{1} \mathrm{H}$ NMR $\left(400 \mathrm{MHz}, \mathrm{CDCl}_{3}, 293 \mathrm{~K}, \mathrm{TMS}\right):$ 7.44-7.60 (5H, m), 7.94-8.01 $(2 \mathrm{H}, \mathrm{m}) ;{ }^{13} \mathrm{C} \mathrm{NMR}\left(100 \mathrm{MHz}, \mathrm{CDCl}_{3}, 293 \mathrm{~K}, \mathrm{TMS}\right): 114.4\left(\mathrm{td}, J_{F}=19.6,3.9 \mathrm{~Hz}\right)$, 123.8, 124.6, 125.2, 126.4, 127.1, 128.7, 129.0, 130.2, 131.6, 133.7, $137.8(\mathrm{dm}$, $\left.J_{F}=253.5 \mathrm{~Hz}\right), 141.0\left(\mathrm{dm}, J_{F}=254.0 \mathrm{~Hz}\right), 144.6\left(\mathrm{dm}, J_{F}=247.4 \mathrm{~Hz}\right), 150.5,150.6$; ${ }^{19} \mathrm{~F}$ NMR $\left(377 \mathrm{MHz}, \mathrm{CDCl}_{3}, 293 \mathrm{~K}, \mathrm{TMS}\right):-144.5\left(2 \mathrm{~F}, \mathrm{dd}, J_{F}=25.4,9.9 \mathrm{~Hz}\right),-159.6$ 
$\left(1 \mathrm{~F}, \mathrm{t}, J_{F}=21.2 \mathrm{~Hz}\right),-166.9\left(2 \mathrm{~F}, \mathrm{ddd}, J_{F}=22.3,20.5,8.7 \mathrm{~Hz}\right)$; HRMS calcd for $\mathrm{C}_{16} \mathrm{H}_{7} \mathrm{~F}_{5}(\mathrm{M}+)$ 294.2188; found: 294.2132 .

\section{9-(perfluorophenyl)phenanthrene (12)}<smiles>Fc1c(F)c(F)c(-c2cc3ccccc3c3ccccc23)c(F)c1F</smiles>

The compound was prepared following the general procedure in $97 \%$ yield: $\mathrm{mp}$ $151-153^{\circ} \mathrm{C}\left(\mathrm{CHCl}_{3}\right) ; \mathrm{R}_{f}=0.30\left(\mathrm{SiO}_{2}, 100 \%\right.$ Hexane$) ; \mathrm{IR}\left(v_{\max } / \mathrm{cm}^{-1}\right): 3067,1520$, 1499, 1490, 988; ${ }^{1} \mathrm{H}$ NMR (400MHz, $\left.\mathrm{CDCl}_{3}, 293 \mathrm{~K}, \mathrm{TMS}\right): 7.51(1 \mathrm{H}, \mathrm{d}, \mathrm{J}=7.5 \mathrm{~Hz})$, $7.58(1 \mathrm{H}, \mathrm{d}, J=7.6 \mathrm{~Hz}), 7.65(1 \mathrm{H}, \mathrm{d}, J=7.3 \mathrm{~Hz}), 7.69-7.75(3 \mathrm{H}, \mathrm{m}), 7.90(1 \mathrm{H}, \mathrm{d}, J=$ $7.8 \mathrm{~Hz}) 8.74(1 \mathrm{H}, \mathrm{d}, J=8.3 \mathrm{~Hz}), 8.78(1 \mathrm{H}, \mathrm{d}, J=8.3 \mathrm{~Hz}) ;{ }^{13} \mathrm{C} \mathrm{NMR}\left(100 \mathrm{MHz}, \mathrm{CDCl}_{3}\right.$, 293K, TMS): $114.4\left(\mathrm{td}, J_{F}=19.5,4.0 \mathrm{~Hz}\right), 122.7,122.7,123.2,125.3,127.2$, $127.2,127.2,127.9,129.0,130.0,130.5,130.7,130.9,130.9,137.8\left(\mathrm{dm}, J_{F}=\right.$ $253.7 \mathrm{~Hz}), 141.0\left(\mathrm{dm}, J_{F}=254.4 \mathrm{~Hz}\right), 144.9\left(\mathrm{dm}, J_{F}=247.5 \mathrm{~Hz}\right) ;{ }^{19} \mathrm{~F} N M R$ (377MHz, $\left.\mathrm{CDCl}_{3}, 293 \mathrm{~K}, \mathrm{TMS}\right):-144.1\left(2 \mathrm{~F}, \mathrm{dd}, J_{F}=24.5,9.3 \mathrm{~Hz}\right),-159.4\left(1 \mathrm{~F}, \mathrm{t}, J_{F}\right.$ $=20.3 \mathrm{~Hz}),-166.7\left(2 \mathrm{~F}, \mathrm{ddd}, \mathrm{J}_{F}=23.4,20.8,8.6 \mathrm{~Hz}\right) ; \mathrm{HRMS}$ calcd for $\mathrm{C}_{20} \mathrm{H}_{9} \mathrm{~F}_{5}(\mathrm{M}+)$ 344.0624; found: 344.0635 .

\section{2',3',4',5',6'-pentamethyl-2,3,4,5,6-pentafluoro-1,1'-biphenyl (13)}<smiles>Cc1c(C)c(C)c(-c2c(F)c(F)c(F)c(F)c2F)c(C)c1C</smiles>

The compound was prepared following the general procedure but using 3.0 eq. of pentafluorobenzene in $85 \%$ yield: $\mathrm{mp} 157-160^{\circ} \mathrm{C}\left(\mathrm{CHCl}_{3}\right) ; \mathrm{R}_{f}=0.42\left(\mathrm{SiO}_{2}\right.$, $100 \%$ pentane); IR $\left(v_{\max } / \mathrm{cm}^{-1}\right): 2950,1492,1120,978 ;{ }^{1} \mathrm{H}$ NMR $(400 \mathrm{MHz}$, 
$\left.\mathrm{CDCl}_{3}, 293 \mathrm{~K}, \mathrm{TMS}\right): 1.99(6 \mathrm{H}, \mathrm{s}), 2.26(6 \mathrm{H}, \mathrm{s}), 2.29(3 \mathrm{H}, \mathrm{s}) ;{ }^{13} \mathrm{C} \mathrm{NMR}(100 \mathrm{MHz}$, $\left.\mathrm{CDCl}_{3}, 293 \mathrm{~K}, \mathrm{TMS}\right): 16.7,17.0,17.9,116.5\left(\mathrm{td}, J_{F}=21.2,3.8 \mathrm{~Hz}\right), 123.2\left(\mathrm{~d}, J_{F}=\right.$ $1.1 \mathrm{~Hz}), 132.6,133.2,136.8,137.8\left(\mathrm{dm}, J_{F}=253.3 \mathrm{~Hz}\right), 140.5\left(\mathrm{dm}, J_{F}=252.6 \mathrm{~Hz}\right)$, $144.0\left(\mathrm{dm}, J_{F}=244.4 \mathrm{~Hz}\right) ;{ }^{19} \mathrm{~F} \mathrm{NMR}\left(377 \mathrm{MHz}, \mathrm{CDCl}_{3}, 293 \mathrm{~K}, \mathrm{TMS}\right):-144.9(2 \mathrm{~F}$, $\left.\mathrm{dd}, J_{F}=23.9,8.7 \mathrm{~Hz}\right),-161.0\left(1 \mathrm{~F}, \mathrm{t}, J_{F}=20.8 \mathrm{~Hz}\right),-167.4\left(2 \mathrm{~F}, \mathrm{ddd}, J_{F}=23.7,20.7\right.$, 8.5Hz); HRMS calcd for $\mathrm{C}_{17} \mathrm{H}_{15} \mathrm{~F}_{5}(\mathrm{M}+)$ 314.1094; found: 314.1114 .

\section{2'-fluoro-2,3,4,5,6-pentafluoro-1,1'-biphenyl (14) ${ }^{9}$}<smiles>Fc1ccccc1-c1c(F)c(F)c(F)c(F)c1F</smiles>

The compound was prepared following the general procedure in $99 \%$ yield: $\mathrm{mp}$ $57-59^{\circ} \mathrm{C}\left(\mathrm{CHCl}_{3}\right) ; \mathrm{R}_{f}=0.44\left(\mathrm{SiO}_{2}, 100 \%\right.$ Hexane$) ; \mathrm{IR}\left(v_{\max } / \mathrm{cm}^{-1}\right): 2939,1488$, 1445, 1231, 1064, 987; ${ }^{1} \mathrm{H}$ NMR $\left(400 \mathrm{MHz}, \mathrm{CDCl}_{3}, 293 \mathrm{~K}, \mathrm{TMS}\right): 7.22$ (1H, ddd, $J=8.5,8.5,1.0 \mathrm{~Hz}), 7.26(1 \mathrm{H}, \mathrm{td}, J=7.6,1.1 \mathrm{~Hz}), 7.35(1 \mathrm{H}, \mathrm{td}, J=6.9,0.6 \mathrm{~Hz}), 7.45-$ 7.51 (1H, dddd, J=15.7, 7.3, 5.3, 1.8Hz); $\left.{ }^{13} \mathrm{C} \mathrm{NMR} \mathrm{(100MHz,} \mathrm{CDCl} 3,293 \mathrm{~K}, \mathrm{TMS}\right)$ : $110.3\left(\mathrm{td}, J_{F}=18.6,4.0 \mathrm{~Hz}\right), 114.4\left(\mathrm{dd}, J_{F}=16.0,1.5 \mathrm{~Hz}\right), 116.3\left(\mathrm{~d}, J_{F}=21.6 \mathrm{~Hz}\right)$, $124.5\left(\mathrm{~d}, J_{F}=3.7 \mathrm{~Hz}\right), 131.8\left(\mathrm{~d}, J_{F}=8.3 \mathrm{~Hz}\right) 132.1\left(\mathrm{~d}, J_{F}=0.8 \mathrm{~Hz}\right), 137.9\left(\mathrm{dm}, J_{F}=\right.$ 252.7Hz), $141.3\left(\mathrm{dm}, J_{F}=254.6 \mathrm{~Hz}\right), 144.5\left(\mathrm{dm}, J_{F}=160.1 \mathrm{~Hz}\right), 160.1\left(\mathrm{~d}, J_{F}=\right.$ 250.7Hz); ${ }^{19} \mathrm{~F} \mathrm{NMR}\left(377 \mathrm{MHz}, \mathrm{CDCl}_{3}, 293 \mathrm{~K}, \mathrm{TMS}\right):-117.9\left(1 \mathrm{~F}, \mathrm{t}, J_{F}=10.9 \mathrm{~Hz}\right)$, $145.4\left(2 \mathrm{~F}, \mathrm{ddd}, J_{F}=24.8,10.6,7.7 \mathrm{~Hz}\right),-159.3\left(1 \mathrm{~F}, \mathrm{t}, J_{F}=21.0 \mathrm{~Hz}\right),-167.2(2 \mathrm{~F}$, ddd, $\left.J_{F}=22.9,20.5,7.8 \mathrm{~Hz}\right)$; HRMS calcd for $\mathrm{C}_{12} \mathrm{H}_{4} \mathrm{~F}_{6}(\mathrm{M}+)$ 262.0217; found: 262.0218 . 


\section{Decafluorobiphenyl (15) ${ }^{10}$}<smiles>Fc1c(F)c(F)c(-c2c(F)c(F)c(F)c(F)c2F)c(F)c1F</smiles>

The compound was prepared following the general procedure but using $3.0 \mathrm{eq}$. of pentafluorobenzene in $83 \%$ yield: $\mathrm{mp} 68-70^{\circ} \mathrm{C}\left(\mathrm{CHCl}_{3}\right) ; \mathrm{R}_{f}=0.55\left(\mathrm{SiO}_{2}, 100 \%\right.$ pentane); IR $\left(v_{\max } / \mathrm{cm}^{-1}\right): 2929,1507,976 ;{ }^{13} \mathrm{C} \mathrm{NMR}\left(100 \mathrm{MHz}, \mathrm{CDCl}_{3}, 293 \mathrm{~K}\right.$, TMS): $101.5-102.0(\mathrm{~m}), 138.2\left(\mathrm{dm}, J_{F}=253.8 \mathrm{~Hz}\right), 142.8\left(\mathrm{dm}, J_{F}=258.1 \mathrm{~Hz}\right)$, $144.8\left(\mathrm{dm}, J_{F}=252.3 \mathrm{~Hz}\right) ;{ }^{19} \mathrm{~F}$ NMR $\left(377 \mathrm{MHz}, \mathrm{CDCl}_{3}, 293 \mathrm{~K}, \mathrm{TMS}\right):-142.6--142.7$ (2F, m), -155.0 (1F, t, $\left.J_{F}=20.7 \mathrm{~Hz}\right),-165.5-165.7(2 \mathrm{~F}, \mathrm{~m})$; HRMS calcd for $\mathrm{C}_{12} \mathrm{~F}_{10}$ $(\mathrm{M}+)$ 333.9840; found: 333.9826 .

\section{4-(perfluorophenyl)acetophenone (21)}<smiles>CC(=O)c1ccc(-c2c(F)c(F)c(F)c(F)c2F)cc1</smiles>

The compound was prepared following the general procedure in $62 \%$ yield: $\mathrm{mp}$ $78-81^{\circ} \mathrm{C}\left(\mathrm{CHCl}_{3}\right) ; \mathrm{R}_{f}=0.29\left(\mathrm{SiO}_{2}, 15 \%\right.$ Ether/Hexane); IR $\left(v_{\max } / \mathrm{cm}^{-1}\right): 2933$, 1688, 1486, 1264, 1062, 982; ${ }^{1} \mathrm{H}$ NMR $\left(400 \mathrm{MHz}, \mathrm{CDCl}_{3}, 293 \mathrm{~K}, \mathrm{TMS}\right): 2.63,(3 \mathrm{H}$, s), $7.52(2 \mathrm{H}, \mathrm{d}, J=8.3 \mathrm{~Hz}), 8.05(2 \mathrm{H}, \mathrm{d}, J=8.4 \mathrm{~Hz}) ;{ }^{13} \mathrm{C} \mathrm{NMR}\left(100 \mathrm{MHz}, \mathrm{CDCl}_{3}\right.$, 293K, TMS): 26.7, 115.0 (td, $\left.J_{F}=16.8,4.1 \mathrm{~Hz}\right), 128.6,130.6,131.1,137.6,138.0$ $\left(\mathrm{dm}, J_{F}=253.3 \mathrm{~Hz}\right), 140.9\left(\mathrm{dm}, J_{F}=255.1 \mathrm{~Hz}\right), 144.1\left(\mathrm{dm}, J_{F}=248.7 \mathrm{~Hz}\right), 197.4$; ${ }^{19} \mathrm{~F}$ NMR $\left(377 \mathrm{MHz}, \mathrm{CDCl}_{3}, 293 \mathrm{~K}, \mathrm{TMS}\right):-148.3\left(2 \mathrm{~F}, \mathrm{dd}, J_{F}=22.9,8.2 \mathrm{~Hz}\right),-161.2$ $\left(1 \mathrm{~F}, \mathrm{t}, J_{F}=20.9 \mathrm{~Hz}\right),-167.5\left(2 \mathrm{~F}, \mathrm{ddd}, J_{F}=22.2,20.4,9.0 \mathrm{~Hz}\right)$; HRMS calcd for $\mathrm{C}_{14} \mathrm{H}_{7} \mathrm{~F}_{5} \mathrm{O}(\mathrm{M}+)$ 286.0417; found: 286.0428 . 
4-(perfluorophenyl)methylbenzoate (22)<smiles>COC(=O)c1ccc(-c2c(F)c(F)c(F)c(F)c2F)cc1</smiles>

The compound was prepared following the general procedure in $99 \%$ yield: $R_{f}=$ $0.26\left(\mathrm{SiO}_{2}, 5 \%\right.$ Ether/Hexane); IR $\left(v_{\max } / \mathrm{cm}^{-1}\right): 2967,1730,1485,1281,1117$, 983; ${ }^{1} \mathrm{H}$ NMR $\left(400 \mathrm{MHz}, \mathrm{CDCl}_{3}, 293 \mathrm{~K}, \mathrm{TMS}\right): 3.96,(3 \mathrm{H}, \mathrm{s}), 7.52(2 \mathrm{H}, \mathrm{d}, \mathrm{J}=$ $8.6 \mathrm{~Hz}), 8.16(2 \mathrm{H}, \mathrm{d}, \mathrm{J}=8.6 \mathrm{~Hz}) ;{ }^{13} \mathrm{C} \mathrm{NMR}\left(100 \mathrm{MHz}, \mathrm{CDCl}_{3}, 293 \mathrm{~K}, \mathrm{TMS}\right): 52.4$, $115.0\left(\mathrm{td}, J_{F}=17.0,4.0 \mathrm{~Hz}\right), 129.9,130.3,131.0,138.0\left(\mathrm{dm}, J_{F}=253.3 \mathrm{~Hz}\right), 140.9$ $\left(\mathrm{dm}, J_{F}=255.1 \mathrm{~Hz}\right), 144.2\left(\mathrm{dm}, J_{F}=248.8 \mathrm{~Hz}\right), 166.4 ;{ }^{19} \mathrm{~F} \mathrm{NMR}\left(377 \mathrm{MHz}, \mathrm{CDCl}_{3}\right.$, 293K, TMS): -147.8 (2F, dd, $\left.J_{F}=23.1,8.4 \mathrm{~Hz}\right),-159.1\left(1 \mathrm{~F}, \mathrm{t}, J_{F}=20.8 \mathrm{~Hz}\right),-166.6$ $\left(2 \mathrm{~F}\right.$, ddd, $J_{F}=23.4,21.8,8.3 \mathrm{~Hz}$ ); HRMS calcd for $\mathrm{C}_{14} \mathrm{H}_{7} \mathrm{~F}_{5} \mathrm{O}_{2}(\mathrm{M}+) 302.0366$; found: 302.0366 .

\section{2-(perfluorophenyl)-4,5,6a,7-tetrahydro-dibenzo[de,g]quinoline-6-carboxylic} acid tert-butyl ester (24)<smiles>CC(C)(C)OC(=O)N1CCc2cc(-c3c(F)c(F)c(F)c(F)c3F)cc3c2C1Cc1ccccc1-3</smiles>

The compound was prepared following the general procedure using 2-chloro4,5,6a,7-tetrahydro-dibenzo[de,g]quinoline-6-carboxylic acid tert-butyl ester ${ }^{11}$ in 99\% yield: $\mathrm{mp} 176-178^{\circ} \mathrm{C}\left(\mathrm{CHCl}_{3}\right) ; \mathrm{R}_{f}=0.16\left(\mathrm{SiO}_{2}, 10 \%\right.$ Ether/Hexane); IR ( $v_{\max }$ $\left./ \mathrm{cm}^{-1}\right): 2976,1693,1522,1498,1411,1170,990 ;{ }^{1} \mathrm{H} \mathrm{NMR}\left(400 \mathrm{MHz}, \mathrm{CDCl}_{3}\right.$, 293K, TMS): $1.52(9 \mathrm{H}, \mathrm{s}), 2.76-3.06(4 \mathrm{H}, \mathrm{m}), 3.14(1 \mathrm{H}, \mathrm{dd}, \mathrm{J}=14.1,3.3 \mathrm{~Hz}), 4.49$ 
$(1 \mathrm{H}, \mathrm{d}, J=6.7 \mathrm{~Hz}), 4.95(1 \mathrm{H}, \mathrm{d}, J=11.8 \mathrm{~Hz}), 7.16(1 \mathrm{H}, \mathrm{s}), 7.24-7.34(3 \mathrm{H}, \mathrm{m}), 7.64$ $(1 \mathrm{H}, \mathrm{s}), 7.74(1 \mathrm{H}, \mathrm{d}, \mathrm{J}=7.5 \mathrm{~Hz}) ;{ }^{13} \mathrm{C} \mathrm{NMR}\left(100 \mathrm{MHz}, \mathrm{CDCl}_{3}, 293 \mathrm{~K}, \mathrm{TMS}\right): 28.6$, $30.5,34.2,38.7,51.6,80.2,115.8\left(\mathrm{td}, J_{F}=17.3,3.8 \mathrm{~Hz}\right), 123.8,124.0,124.9$, $127.5,128.5,128.8,129.2,133.2,134.1,135.1,135.8,135.9,137.9\left(\mathrm{dm}, J_{F}=\right.$ 253.4Hz), $140.5\left(\mathrm{dm}, J_{F}=249.4 \mathrm{~Hz}\right), 144.3\left(\mathrm{dm}, J_{F}=247.4 \mathrm{~Hz}\right), 154.6 ;{ }^{19} \mathrm{~F} \mathrm{NMR}$ (377MHz, $\left.\mathrm{CDCl}_{3}, 293 \mathrm{~K}, \mathrm{TMS}\right):-147.8\left(2 \mathrm{~F}, \mathrm{dd}, J_{F}=22.9,8.1 \mathrm{~Hz}\right),-160.5\left(1 \mathrm{~F}, \mathrm{t}, J_{F}\right.$ $=21.0 \mathrm{~Hz}),-167.0\left(2 \mathrm{~F}, \mathrm{ddd}, \mathrm{J}_{F}=22.7,21.6,7.8 \mathrm{~Hz}\right) ; \mathrm{HRMS}$ calcd for $\mathrm{C}_{23} \mathrm{H}_{13} \mathrm{~F}_{5} \mathrm{NO}_{2}$ $\left(\mathrm{M}+-\mathrm{C}_{4} \mathrm{H}_{9}\right)$ 430.0866; found: 430.0858 .

\section{3-(perfluorophenyl)quinoline (26)}<smiles>Fc1c(F)c(F)c(-c2cnc3ccccc3c2)c(F)c1F</smiles>

The compound was prepared following the general procedure in $92 \%$ yield: $\mathrm{mp}$ $135-137^{\circ} \mathrm{C}\left(\mathrm{CHCl}_{3}\right) ; \mathrm{R}_{f}=0.17\left(\mathrm{SiO}_{2}, 10 \%\right.$ Ether/Hexane); IR $\left(v_{\max } / \mathrm{cm}^{-1}\right): 2933$, 1500, 1365, 1064, 990; ${ }^{1} \mathrm{H}$ NMR (400MHz, $\left.\mathrm{CDCl}_{3}, 293 \mathrm{~K}, \mathrm{TMS}\right): 7.63(1 \mathrm{H}, \mathrm{t}, \mathrm{J}=$ $7.4 \mathrm{~Hz}), 7.82(1 \mathrm{H}, \mathrm{td}, J=7.2,0.9 \mathrm{~Hz}), 7.89(1 \mathrm{H}, \mathrm{d}, J=8.1 \mathrm{~Hz}), 8.18(1 \mathrm{H}, \mathrm{d}, J=$ 8.5Hz), $8.28(1 \mathrm{H}, \mathrm{s}), 8.95(1 \mathrm{H}, \mathrm{s}) ;{ }^{13} \mathrm{C} \mathrm{NMR}\left(100 \mathrm{MHz}, \mathrm{CDCl}_{3}, 293 \mathrm{~K}, \mathrm{TMS}\right): 112.8$ (dt, $\left.J_{F}=17.2,4.0 \mathrm{~Hz}\right), 120.1,127.4,127.6,128.2,129.5,130.9,137.8,138.1(\mathrm{dm}$, $\left.J_{F}=253.8 \mathrm{~Hz}\right), 141.1\left(\mathrm{dm}, J_{F}=255.6 \mathrm{~Hz}\right), 144.5\left(\mathrm{dm}, J_{F}=248.9 \mathrm{~Hz}\right), 147.9 ;{ }^{19} \mathrm{~F}$ NMR $\left(377 \mathrm{MHz}, \mathrm{CDCl}_{3}, 293 \mathrm{~K}, \mathrm{TMS}\right):-147.8\left(2 \mathrm{~F}, \mathrm{dd}, J_{F}=22.7,8.3 \mathrm{~Hz}\right),-158.4$ $\left(1 \mathrm{~F}, \mathrm{t}, J_{F}=20.7 \mathrm{~Hz}\right),-166.1\left(2 \mathrm{~F}, \mathrm{ddd}, J_{F}=22.9,20.5,7.1 \mathrm{~Hz}\right)$; HRMS calcd for $\mathrm{C}_{15} \mathrm{H}_{6} \mathrm{NF}_{5}(\mathrm{M}+)$ 295.0420; found: 295.0428. 


\section{2,3,5,6-Tetrafluoro-4,2'-dimethyl-biphenyl (30)}<smiles>Cc1ccccc1-c1c(F)c(F)c(C)c(F)c1F</smiles>

The compound was prepared following the general procedure but using 3.0 eq. of pentafluorobenzene in $96 \%$ yield: $\mathrm{R}_{f}=0.43\left(\mathrm{SiO}_{2}, 100 \%\right.$ Hexane); IR ( $v_{\max }$ $\left./ \mathrm{cm}^{-1}\right)$ : 2932, 1481, 1303, 1065, 923; ${ }^{1} \mathrm{H}$ NMR (400MHz, $\left.\mathrm{CDCl}_{3}, 293 \mathrm{~K}, \mathrm{TMS}\right)$ : $2.19(3 \mathrm{H}, \mathrm{s}), 2.3(3 \mathrm{H}, \mathrm{t}, J=2.1 \mathrm{~Hz}), 7.20(1 \mathrm{H}, \mathrm{d}, J=7.4 \mathrm{~Hz}), 7.27(1 \mathrm{H}, \mathrm{tdd}, J=6.5$, 2.2, 0.6Hz), 7.31-7.38 (2H, m); ${ }^{13} \mathrm{C}$ NMR $\left(100 \mathrm{MHz}, \mathrm{CDCl}_{3}, 293 \mathrm{~K}, \mathrm{TMS}\right):$ 7.6-7.7 $(\mathrm{m}), 19.7\left(\mathrm{t}, J_{F}=1.2 \mathrm{~Hz}\right), 115.3\left(\mathrm{t}, J_{F}=19.2 \mathrm{~Hz}\right), 117.6\left(\mathrm{t}, J_{F}=19.5 \mathrm{~Hz}\right), 125.8$, $127.2\left(\mathrm{t}, J_{F}=2.1 \mathrm{~Hz}\right), 129.3,130.4,130.6,137.4,143.6$ (dddd, $J_{F}=244.2,14.3$, $5.9,3.8 \mathrm{~Hz}$ ), 145.1 (dddd, $\left.J_{F}=244.3,14.5,7.3,4.0 \mathrm{~Hz}\right) ;{ }^{19} \mathrm{~F}$ NMR $(377 \mathrm{MHz}$, $\left.\mathrm{CDCl}_{3}, 293 \mathrm{~K}, \mathrm{TMS}\right):-143.0\left(2 \mathrm{~F}, \mathrm{dd}, J_{F}=23.1,13.4 \mathrm{~Hz}\right),-144.2\left(2 \mathrm{~F}, \mathrm{dd}, J_{F}=23.1\right.$, 12.4Hz); HRMS calcd for $\mathrm{C}_{14} \mathrm{H}_{10} \mathrm{~F}_{4}(\mathrm{M}+)$ 254.0719; found: 254.0707 .

\section{2,3,5,6-Tetrafluoro-4-methoxy-4'-methyl-biphenyl (31)}<smiles>COc1c(F)c(F)c(-c2ccccc2C)c(F)c1F</smiles>

The compound was prepared following the general procedure in $94 \%$ yield: $R_{f}=$ $0.33\left(\mathrm{SiO}_{2}, 100 \%\right.$ Hexane); IR $\left(v_{\max } / \mathrm{cm}^{-1}\right): 2959,1485,1123,1082,990 ;{ }^{1} \mathrm{H}$ NMR $\left(400 \mathrm{MHz}, \mathrm{CDCl}_{3}, 293 \mathrm{~K}, \mathrm{TMS}\right): 2.19(3 \mathrm{H}, \mathrm{s}), 4.13(3 \mathrm{H}, \mathrm{t}, \mathrm{J}=1.3 \mathrm{~Hz}), 7.19$ $(1 \mathrm{H}, \mathrm{d}, J=7.7 \mathrm{~Hz}), 7.27(1 \mathrm{H}, \mathrm{tdd}, J=6.8,2.1,0.6 \mathrm{~Hz}), 7.32-7.38(2 \mathrm{H}, \mathrm{m}) ;{ }^{13} \mathrm{C} \mathrm{NMR}$ $\left(100 \mathrm{MHz}, \mathrm{CDCl}_{3}, 293 \mathrm{~K}, \mathrm{TMS}\right): 19.7,62.2,113.8$ (t, $\left.J_{F}=19.9 \mathrm{~Hz}\right), 125.9,126.7$, $129.3,130.4,130.8,137.6,137.7\left(\mathrm{dt}, J_{F}=12.0,3.6 \mathrm{~Hz}\right), 141.1$ (ddt, $J_{F}=247.4$, $15.8,4.3 \mathrm{~Hz}), 144.2$ (dddd, $\left.J_{F}=244.7,12.1,7.9,3.8 \mathrm{~Hz}\right) ;{ }^{19} \mathrm{~F} \mathrm{NMR}(377 \mathrm{MHz}$, $\left.\mathrm{CDCl}_{3}, 293 \mathrm{~K}, \mathrm{TMS}\right):-163.5\left(2 \mathrm{~F}, \mathrm{dd}, J_{F}=22.4,8.9 \mathrm{~Hz}\right),-147.7\left(2 \mathrm{~F}, \mathrm{dd}, J_{F}=22.4\right.$, 8.8Hz); HRMS calcd for $\mathrm{C}_{14} \mathrm{H}_{10} \mathrm{OF}_{4}(\mathrm{M}+)$ 270.0668; found: 270.0689 . 


\section{2,3,5,6-Tetrafluoro-4-o-tolyl-pyridine (32)}<smiles>Cc1ccccc1-c1c(F)c(F)nc(F)c1F</smiles>

The compound was prepared following the general procedure in $97 \%$ yield: $\mathrm{mp}$ $55-57^{\circ} \mathrm{C}\left(\mathrm{CHCl}_{3}\right) ; \mathrm{R}_{f}=0.36\left(\mathrm{SiO}_{2}, 100 \%\right.$ Hexane$) ; \mathrm{IR}\left(v_{\max } / \mathrm{cm}^{-1}\right): 2967,1448$, 1278, 967; ${ }^{1} \mathrm{H}$ NMR (400MHz, CDCl 3 , 293K, TMS): $2.23(3 \mathrm{H}, \mathrm{s}), 7.22(1 \mathrm{H}, \mathrm{d}, \mathrm{J}=$ $7.6 \mathrm{~Hz}), 7.27(1 \mathrm{H}, \mathrm{dt}, J=7.7,0.4 \mathrm{~Hz}), 7.38(1 \mathrm{H}, \mathrm{dd}, J=7.0,0.6 \mathrm{~Hz}), 7.44(1 \mathrm{H}, \mathrm{dt}, J=$ 7.5, 1.1Hz); ${ }^{13} \mathrm{C} \mathrm{NMR}\left(100 \mathrm{MHz}, \mathrm{CDCl}_{3}, 293 \mathrm{~K}, \mathrm{TMS}\right): 19.6,125.4\left(\mathrm{t}, J_{F}=1.9 \mathrm{~Hz}\right)$, $126.2,129.7,130.5,130.8,134.0\left(\mathrm{tt}, J_{F}=17.6,2.9 \mathrm{~Hz}\right), 136.8,139.4$ (ddt, $J_{F}=$ 257.6, 21.1, 6.2Hz), 143.7 (dddd, $J_{F}=245.7,17.6,13.2,2.6 \mathrm{~Hz}$ ); ${ }^{19} \mathrm{~F} N M R$ (377MHz, $\left.\mathrm{CDCl}_{3}, 293 \mathrm{~K}, \mathrm{TMS}\right):-95.9-96.1$ (2F, m), -147.2-147.4 (2F, m); HRMS calcd for $\mathrm{C}_{12} \mathrm{H}_{7} \mathrm{NF}_{4}(\mathrm{M}+)$ 241.0515; found: 241.0493 .

\section{2,4,6-Trifluoro-2'-methyl-biphenyl (33)}<smiles>Cc1ccccc1-c1c(F)cc(F)cc1F</smiles>

The compound was prepared following the general procedure but using $5.0 \mathrm{eq}$. of pentafluorobenzene in 82\% yield: $\mathrm{R}_{f}=0.42\left(\mathrm{SiO}_{2}, 100 \%\right.$ pentane $)$; $\mathrm{mp}$ 37$38^{\circ} \mathrm{C}\left(\mathrm{CHCl}_{3}\right) ; \mathrm{IR}\left(v_{\max } / \mathrm{cm}^{-1}\right): 3066,1597,1475,1119,998 ;{ }^{1} \mathrm{H}$ NMR $(400 \mathrm{MHz}$, $\left.\mathrm{CDCl}_{3}, 293 \mathrm{~K}, \mathrm{TMS}\right): 6.71-6.76(2 \mathrm{H}, \mathrm{m}), 7.18(1 \mathrm{H}, \mathrm{d}, J=7.3 \mathrm{~Hz}), 7.26(1 \mathrm{H}, \mathrm{td}, J=$ 7.9, 2.6Hz), 7.30-7.32 (2H, m); $\left.{ }^{13} \mathrm{C} \mathrm{NMR} \mathrm{(100MHz,} \mathrm{CDCl}_{3}, 293 \mathrm{~K}, \mathrm{TMS}\right): 19.7$, 99.9-100.5 (m), 114.3-114.8 (m), 125.7, 128.0, 128.9, 130.2, 130.8, 137.5, 160.3 $\left(\mathrm{dt}, J_{F}=248.4,12.6 \mathrm{~Hz}\right), 162.1\left(\mathrm{dt}, J_{F}=249.1,15.5 \mathrm{~Hz}\right) ;{ }^{19} \mathrm{~F} \mathrm{NMR}(377 \mathrm{MHz}$, $\mathrm{CDCl}_{3}, 293 \mathrm{~K}, \mathrm{TMS}$ ): -114.1 (3F, broad s); HRMS calcd for $\mathrm{C}_{13} \mathrm{H}_{9} \mathrm{~F}_{3}(\mathrm{M}+)$ 222.0656; found: 222.0650 .

\footnotetext{
${ }^{1}$ Korenaga, T.; Kosaki, T.; Fukumura, R.; Ema, T. ; Sakai, T. Org Lett 2005, 4915.
} 
${ }^{2}$ Chen, Q.-Y. and Li, Z.-T. J. Org. Chem. 1993, 2599.

${ }^{3}$ Kamigata, N.; Yoshikawa, M.; Shimizu, T. J. Fluorine Chem. 1998, 91.

${ }^{4}$ Molander, G. A. and Biolatto, B. . J. Org. Chem. 2003, 4302.

${ }^{5}$ Altenhoff, G.; Goddard, R.; Lehmann, C. W.; Glorius, F. J. Am. Chem. Soc. 2004, 15195.

${ }^{6}$ Lafrance, M.; Rowley, C. N.; Woo, T. K.; Fagnou, K. J. Am. Chem. Soc. 2006, 8754.

${ }^{7}$ Kasemann, R. and Naumann, D. J. Fluorine Chem. 1990, 207.

${ }^{8}$ Birchall, J. M.; Evans, L. R.; Haszeldine, R.N.; J. Chem. Soc. Perkin Trans. 1; 1974, 1715.

${ }^{9}$ Frohn, H.J.; Klose, A.; Bardin, V.V. J. Fluorine Chem. 1993, 201.

${ }^{10}$ Von Fircks, G.; Hausmann, H.; Francke, V.; Gunther, H. J. Org. Chem. 1997, 5074.

${ }^{11}$ Lafrance, M.; Blaquiere N.; Fagnou, K. Eur. J. Org. Chem. In press. 
S13
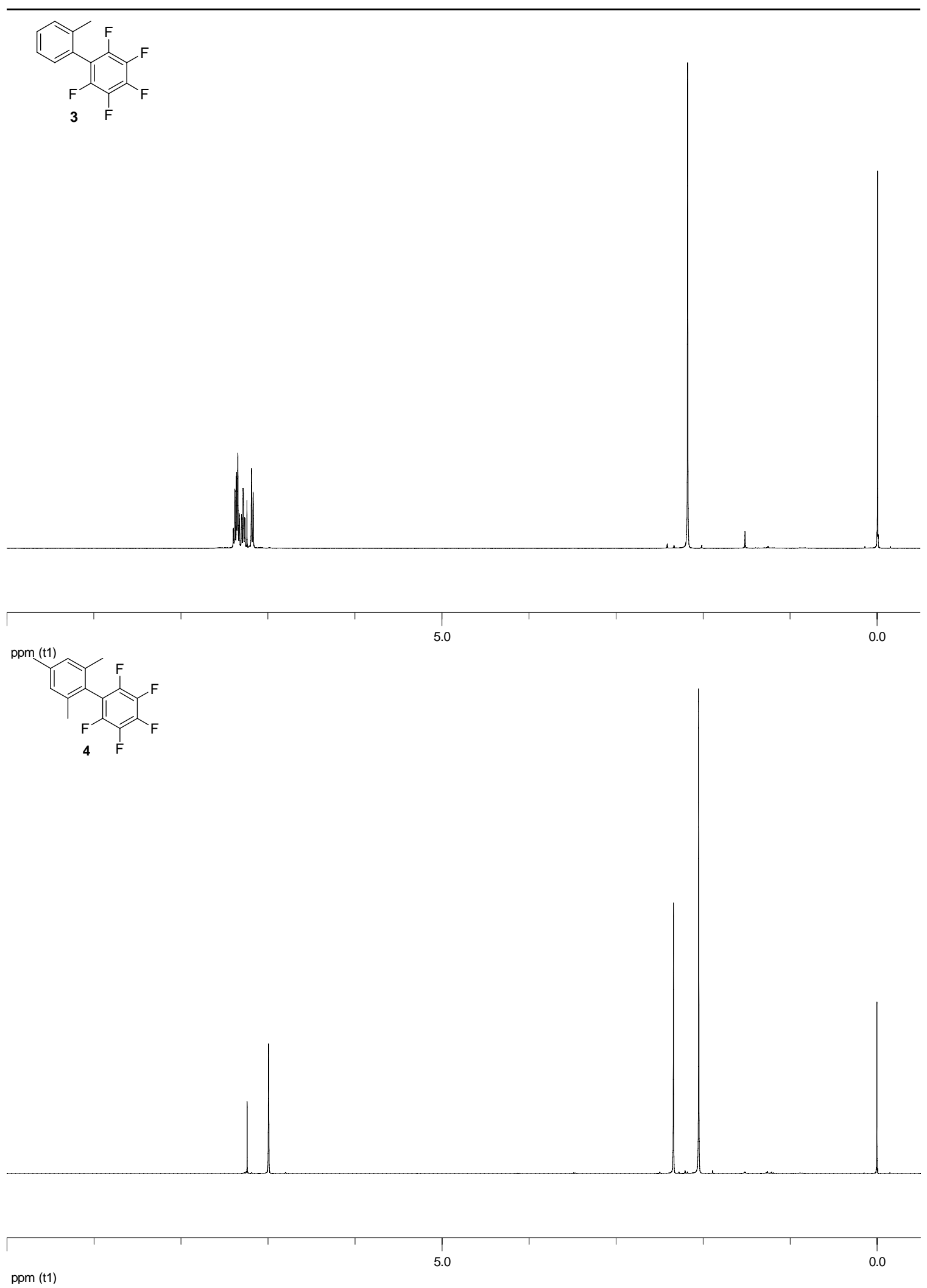
S14
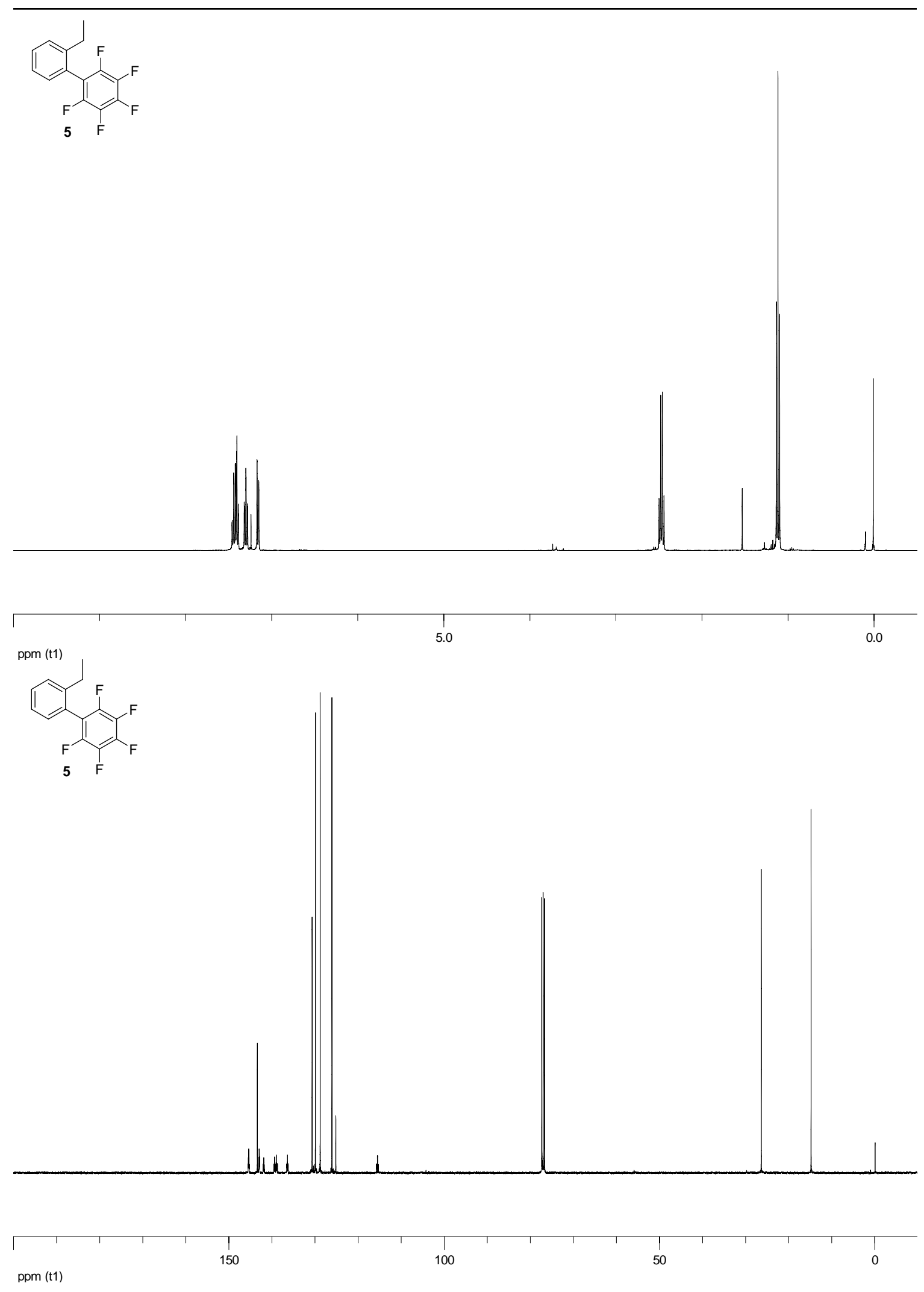

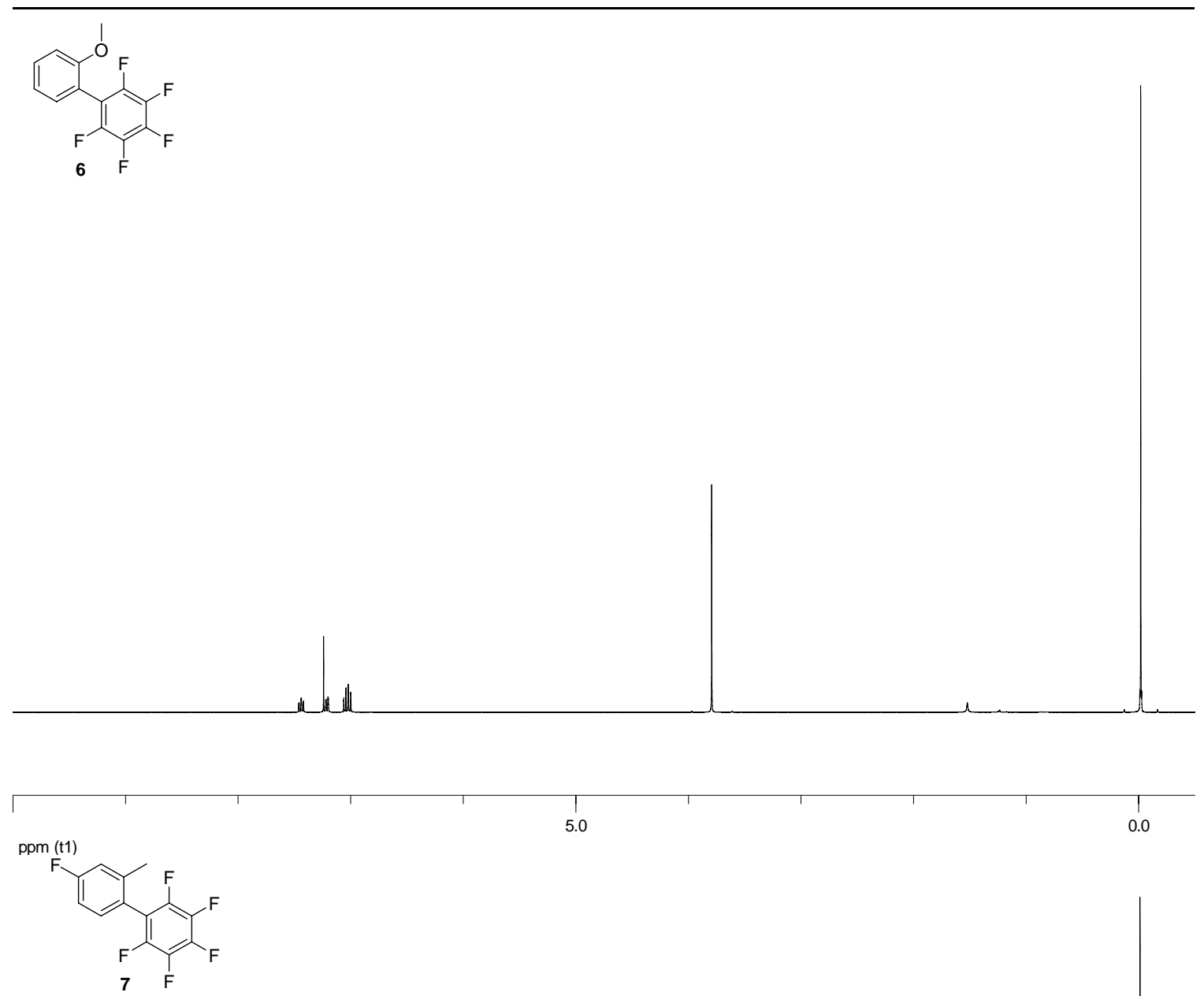

III.
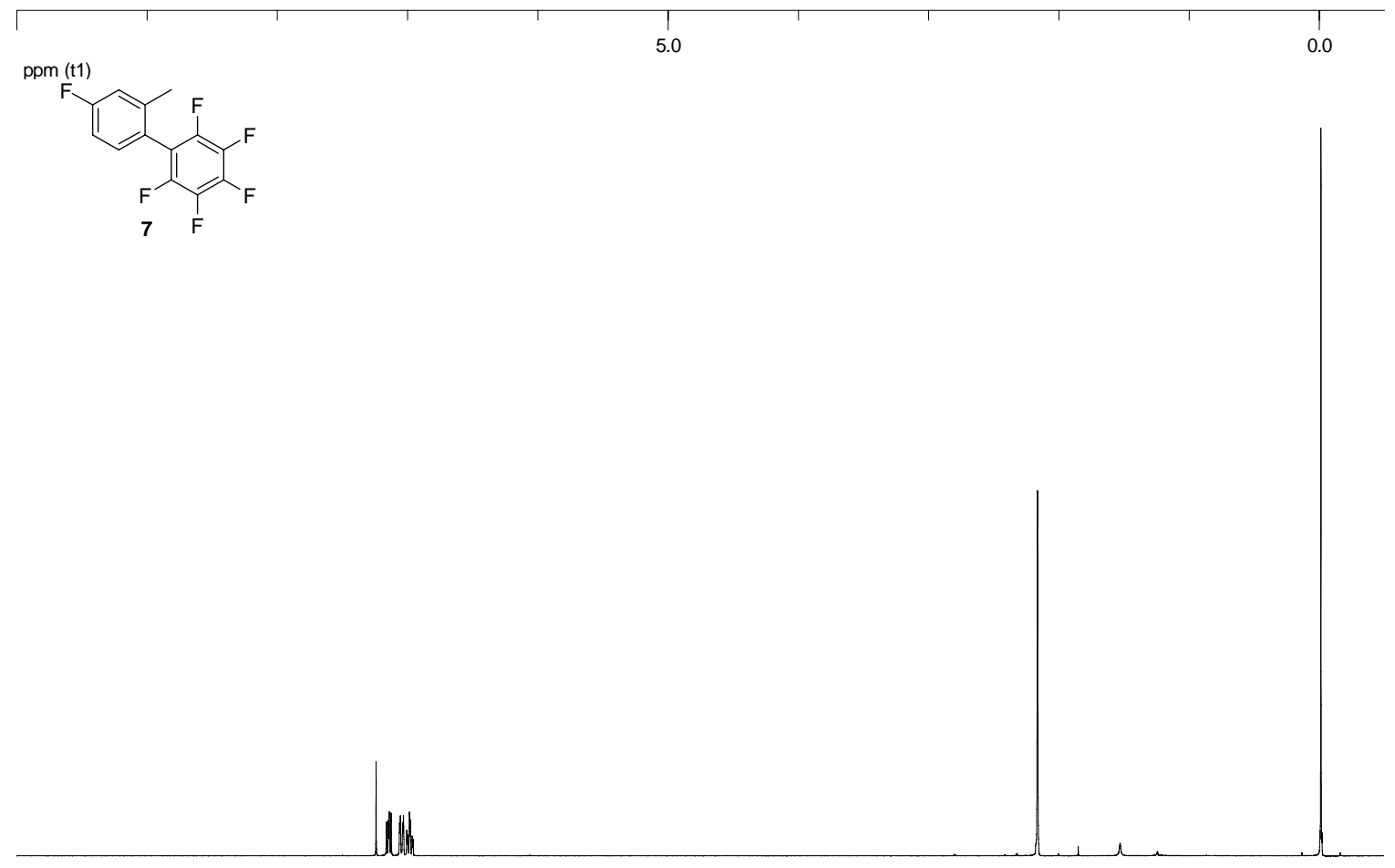

\begin{tabular}{|c|c|c|c|}
\hline 1 & 5.0 \\
& &
\end{tabular}

ppm (t1) 

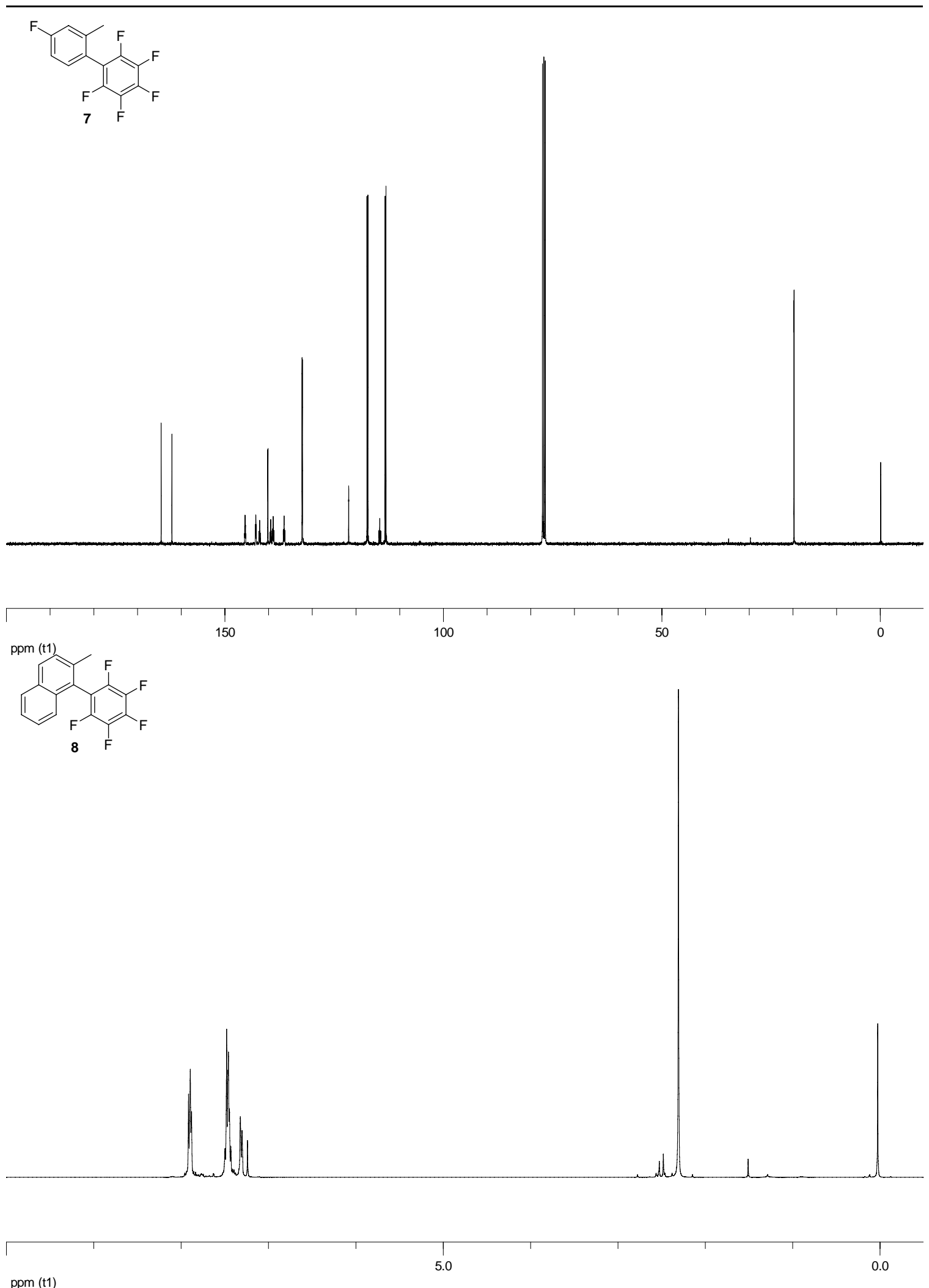

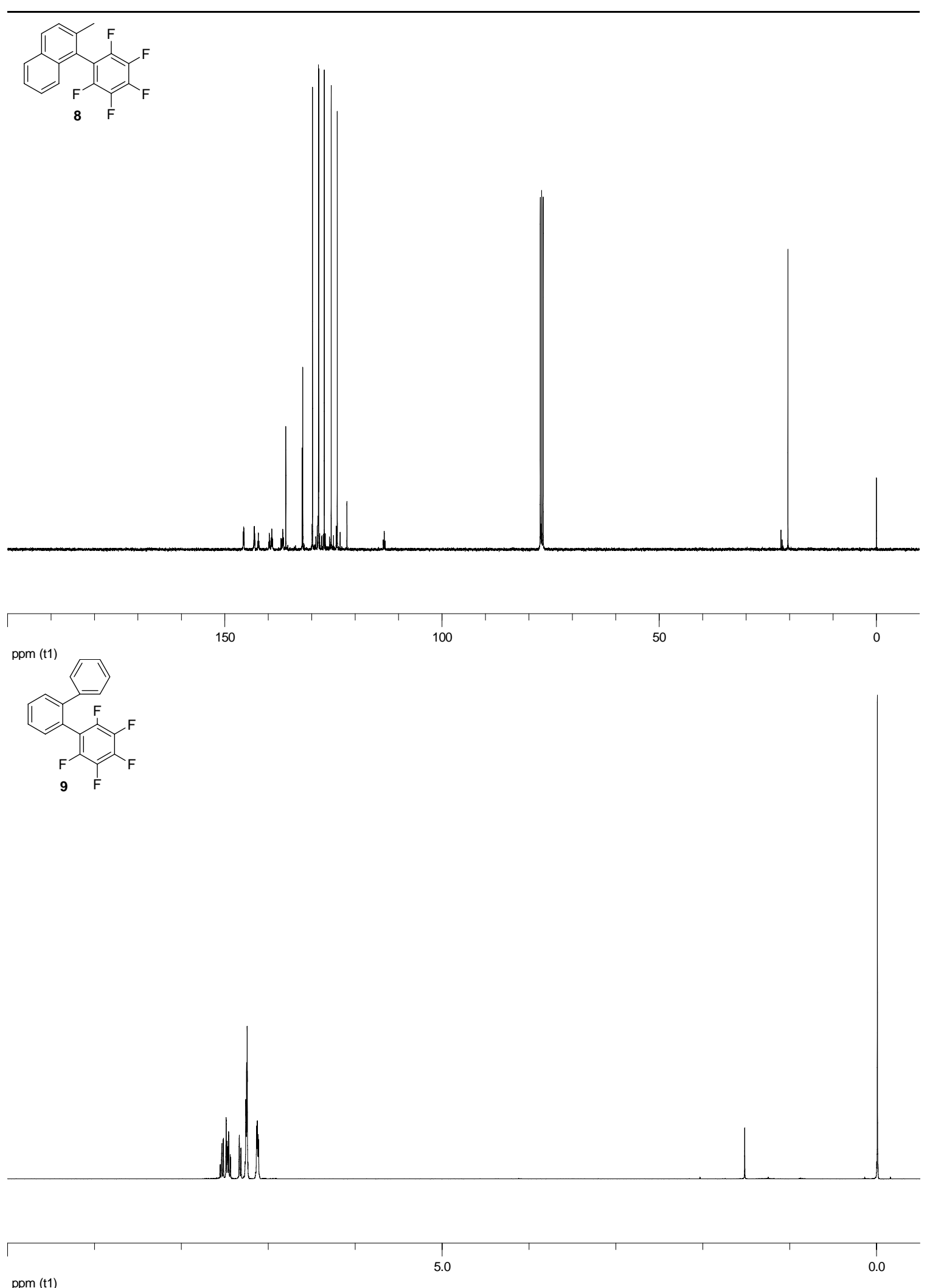

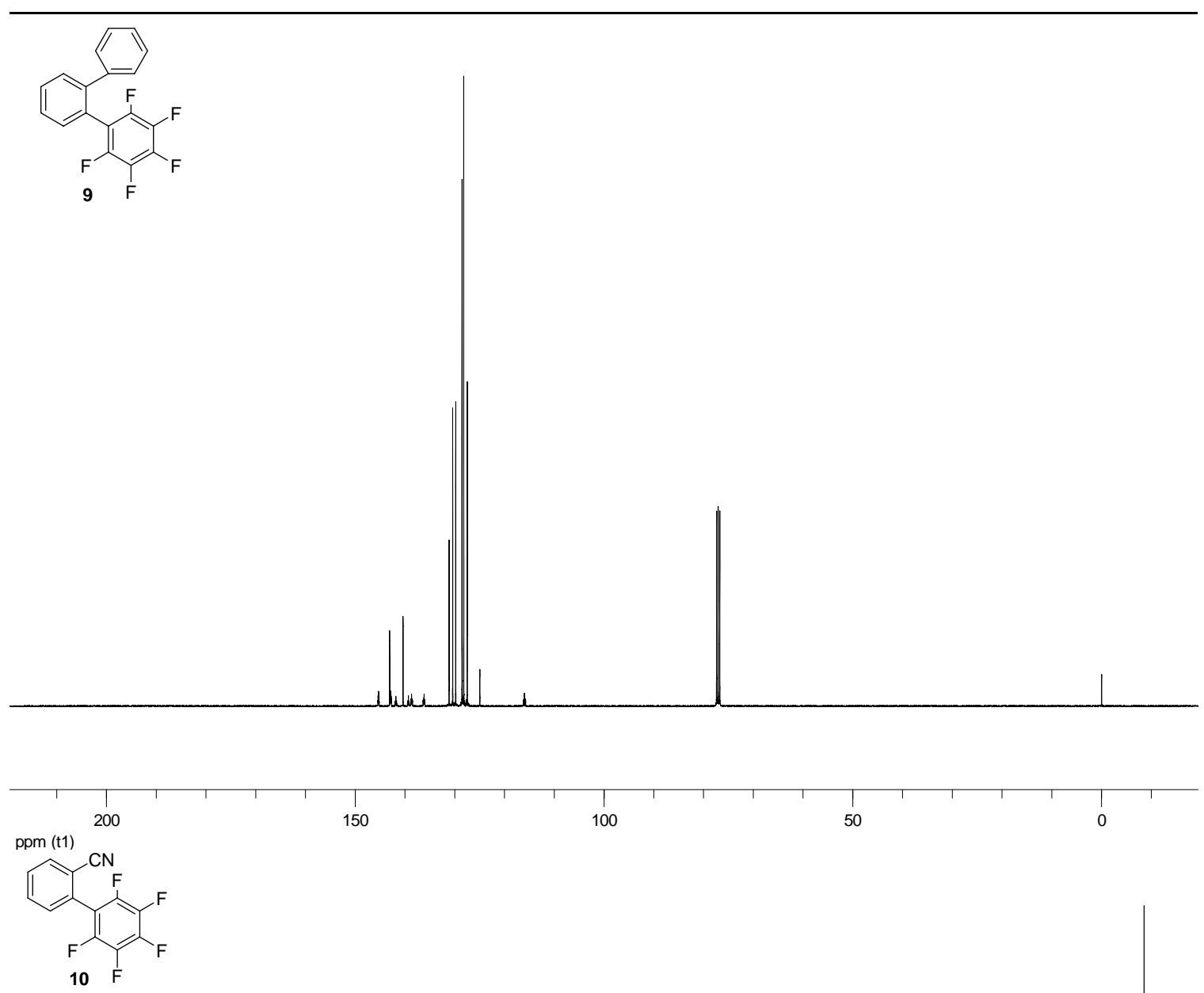

100

I I 10

150
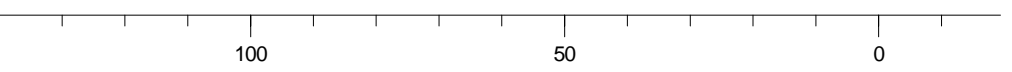

H. H.
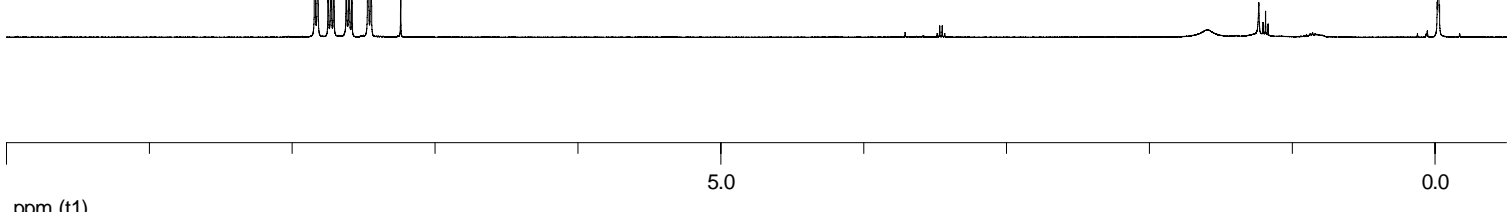

ppm (t1) 

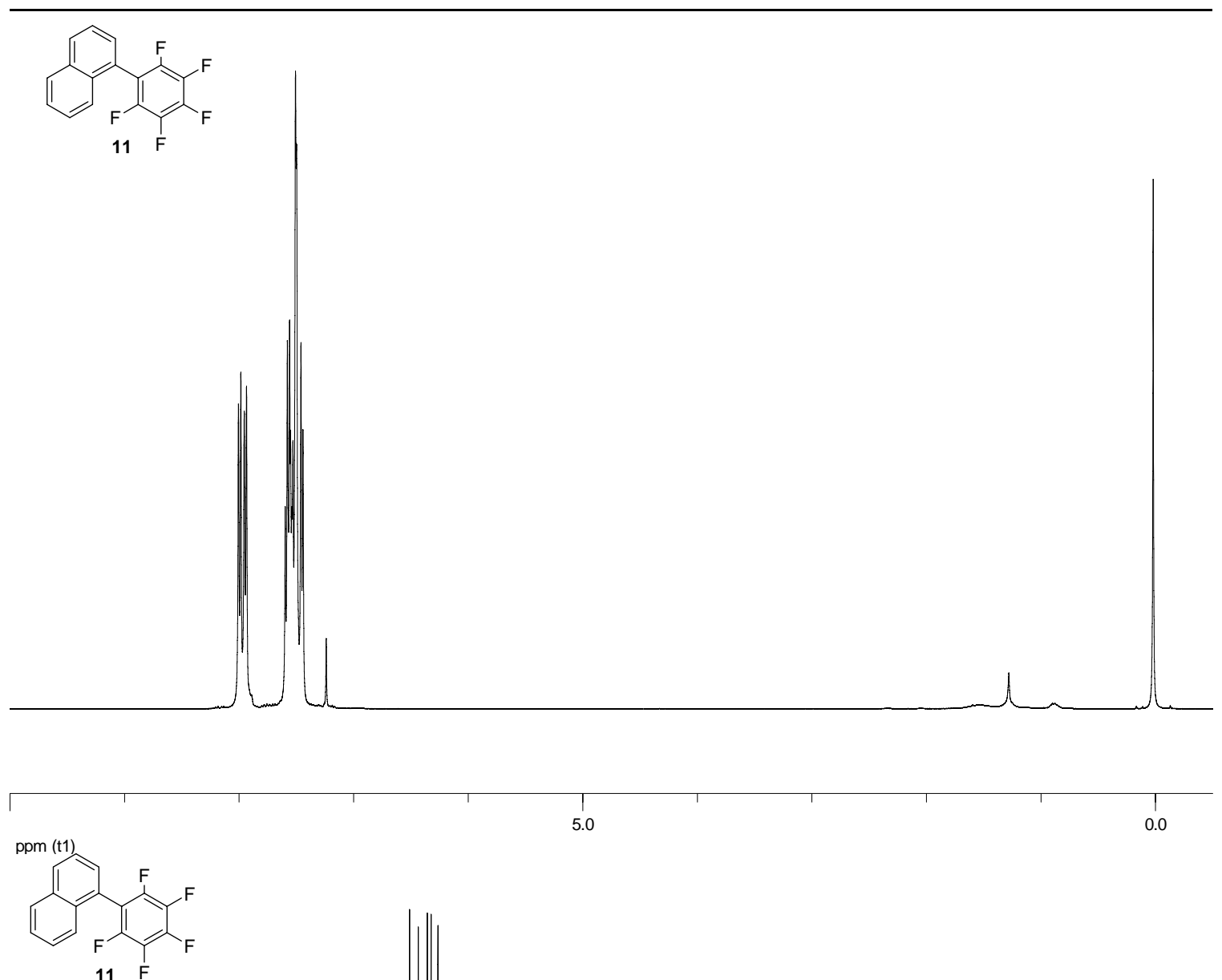

ل

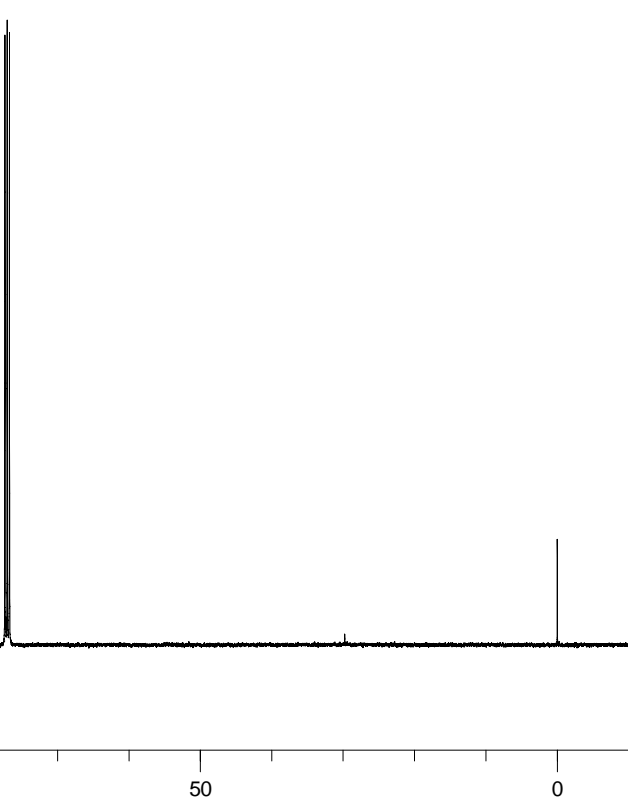

ppm (t1)

150

100 

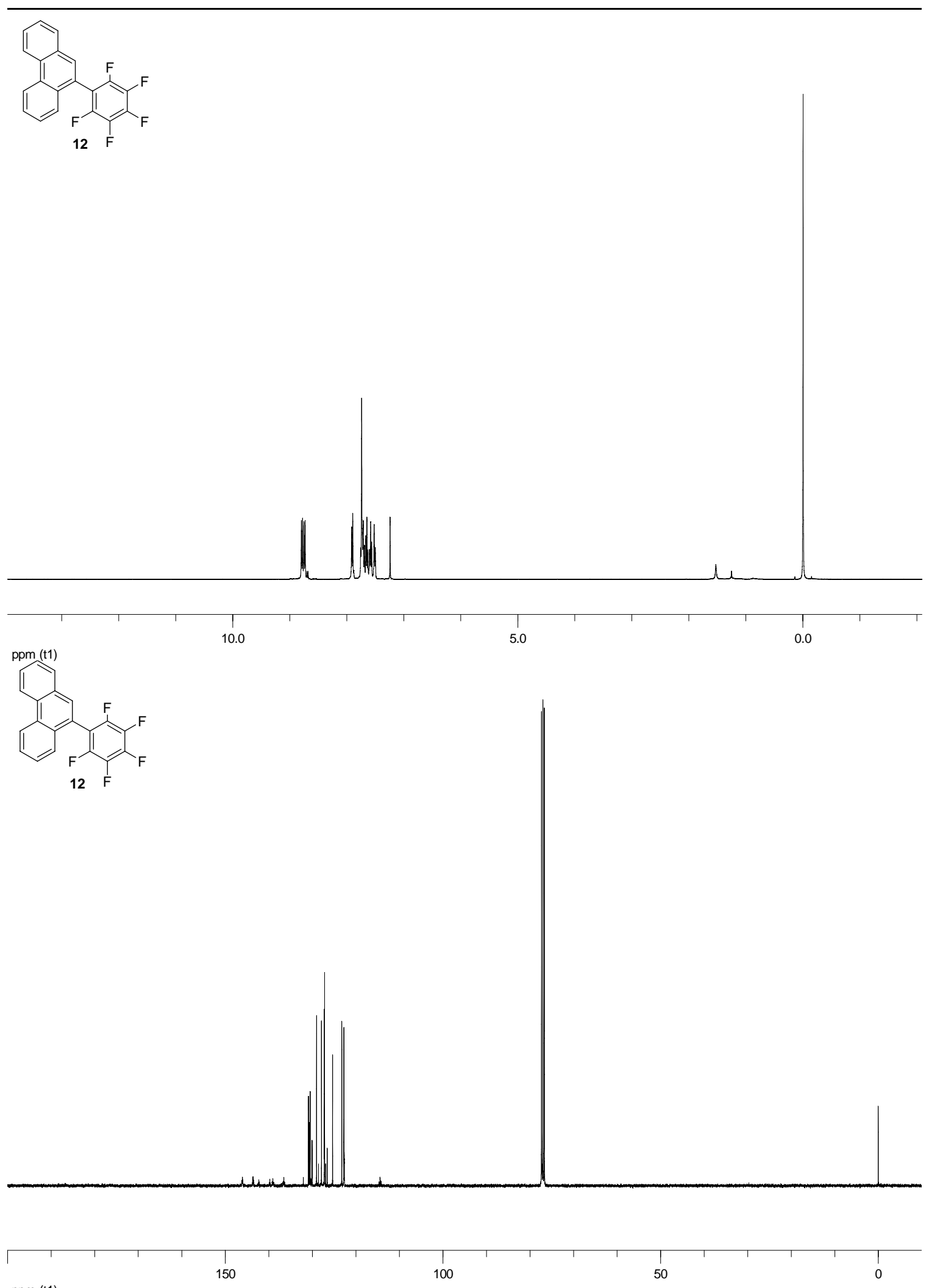

ppm (t1) 

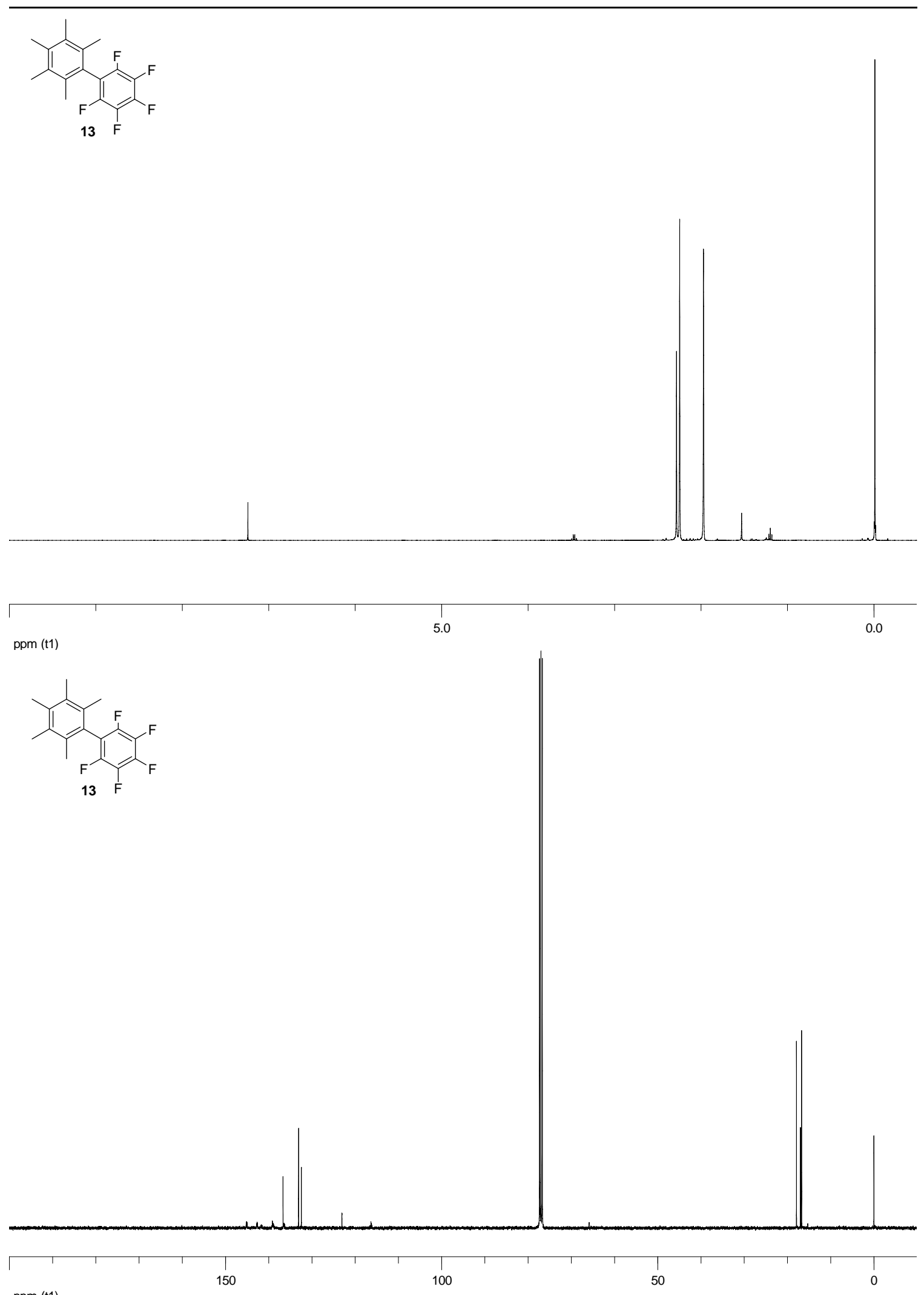

ppm (t1) 

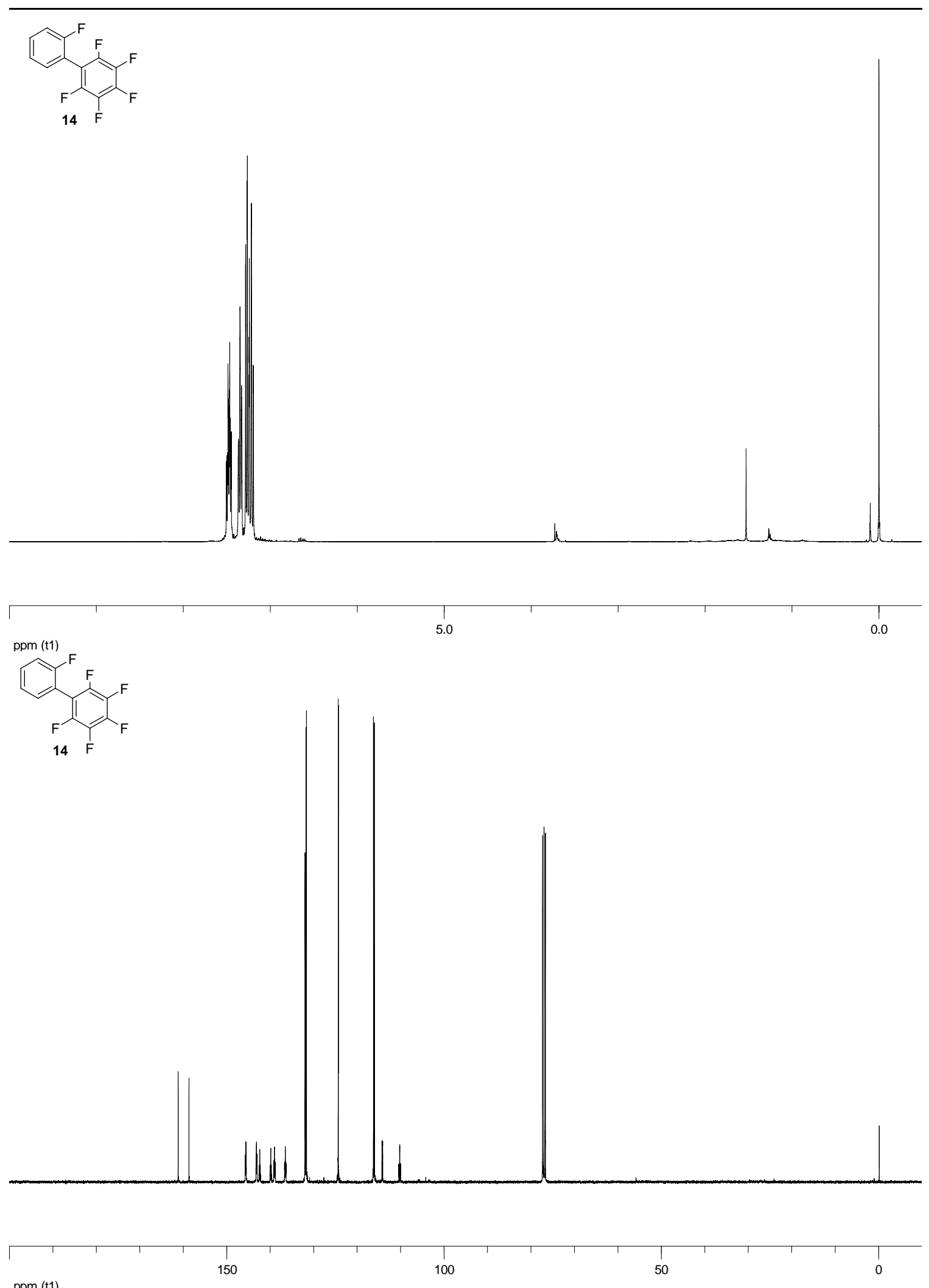

ppm (t1) 

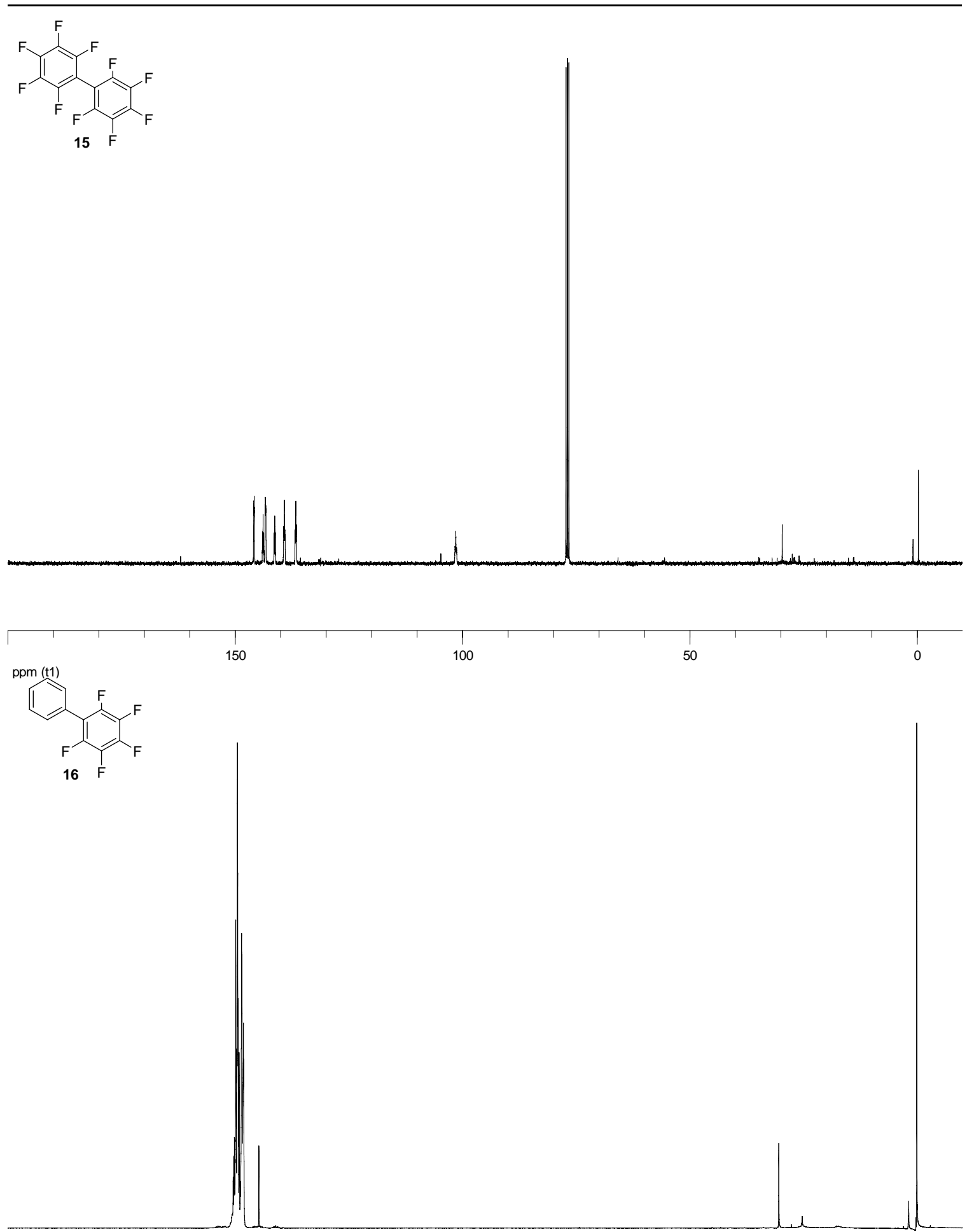

ppm (t1)

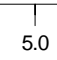

0.0

ppm (I) 

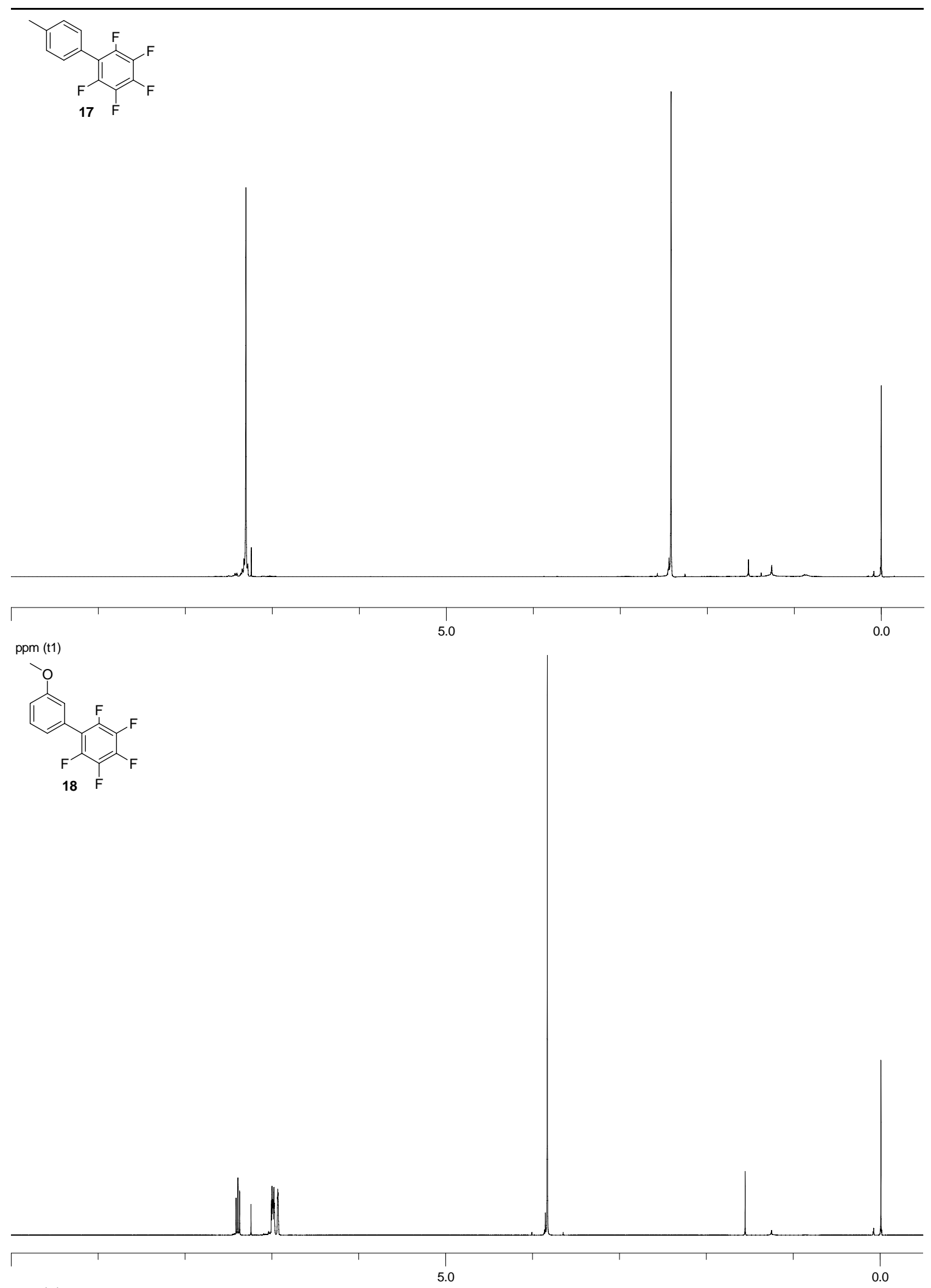

ppm (t1) 

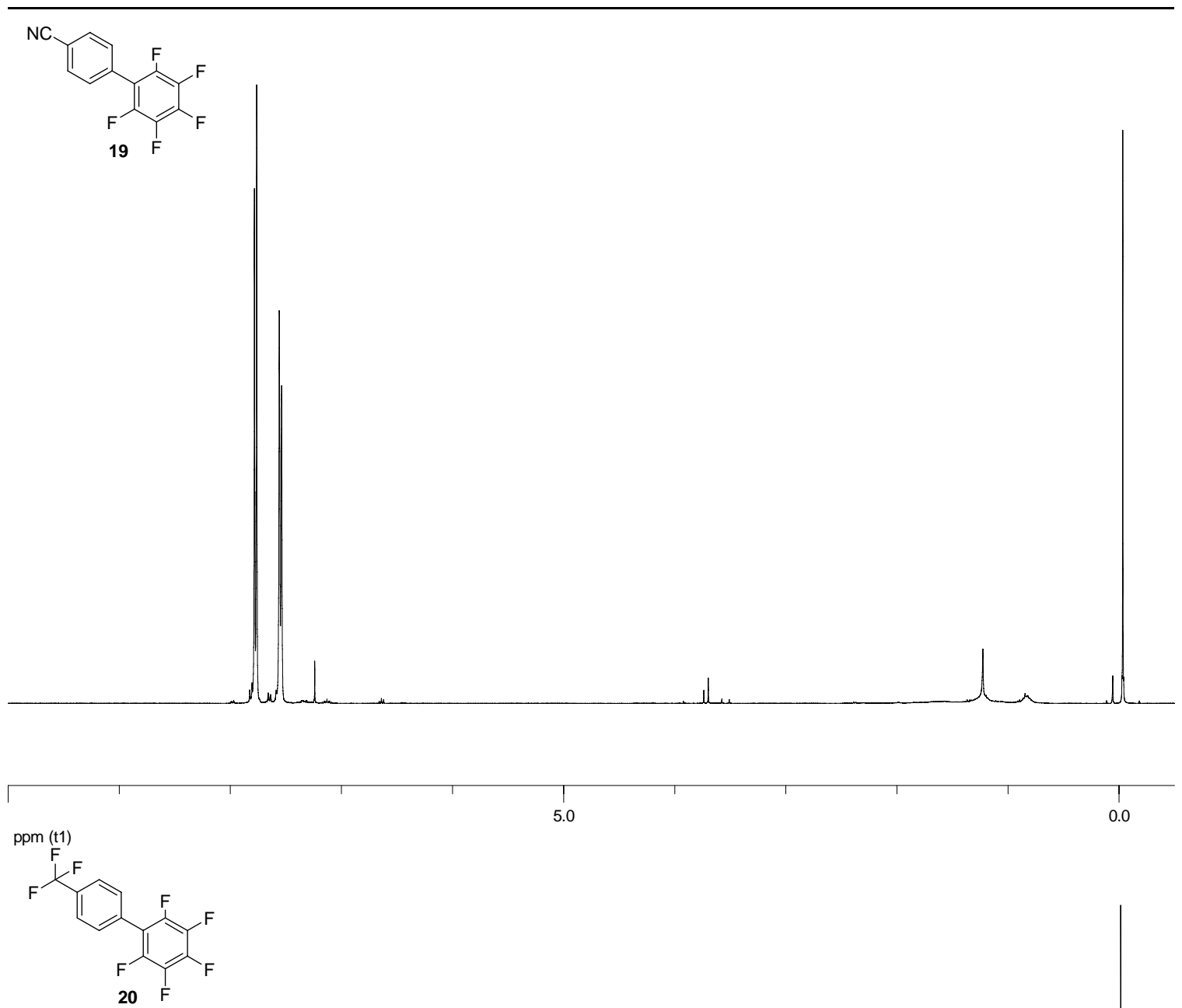

NW
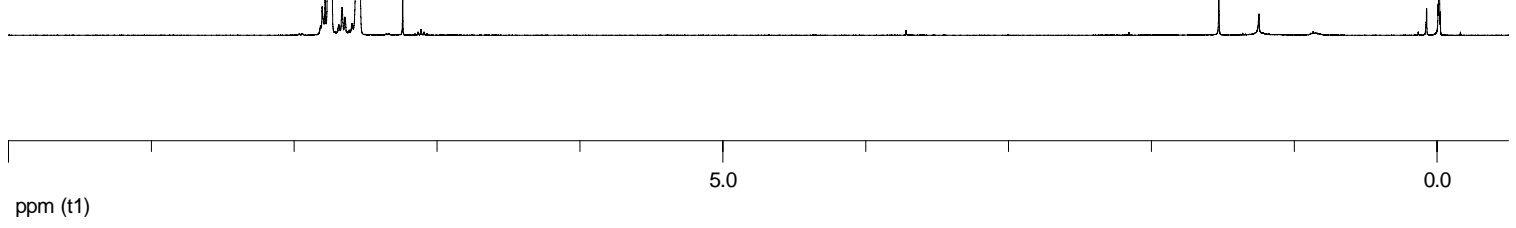
S26
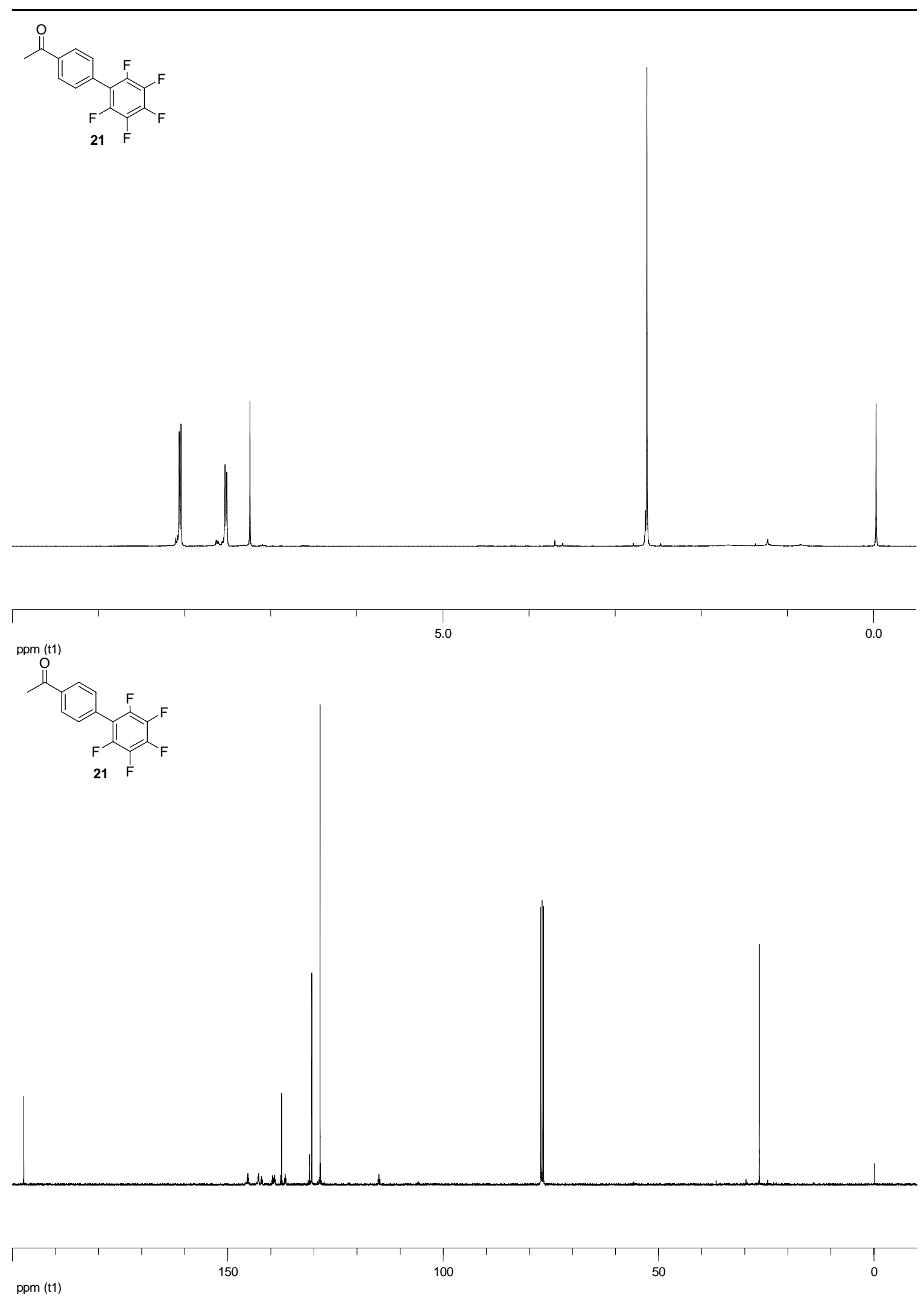

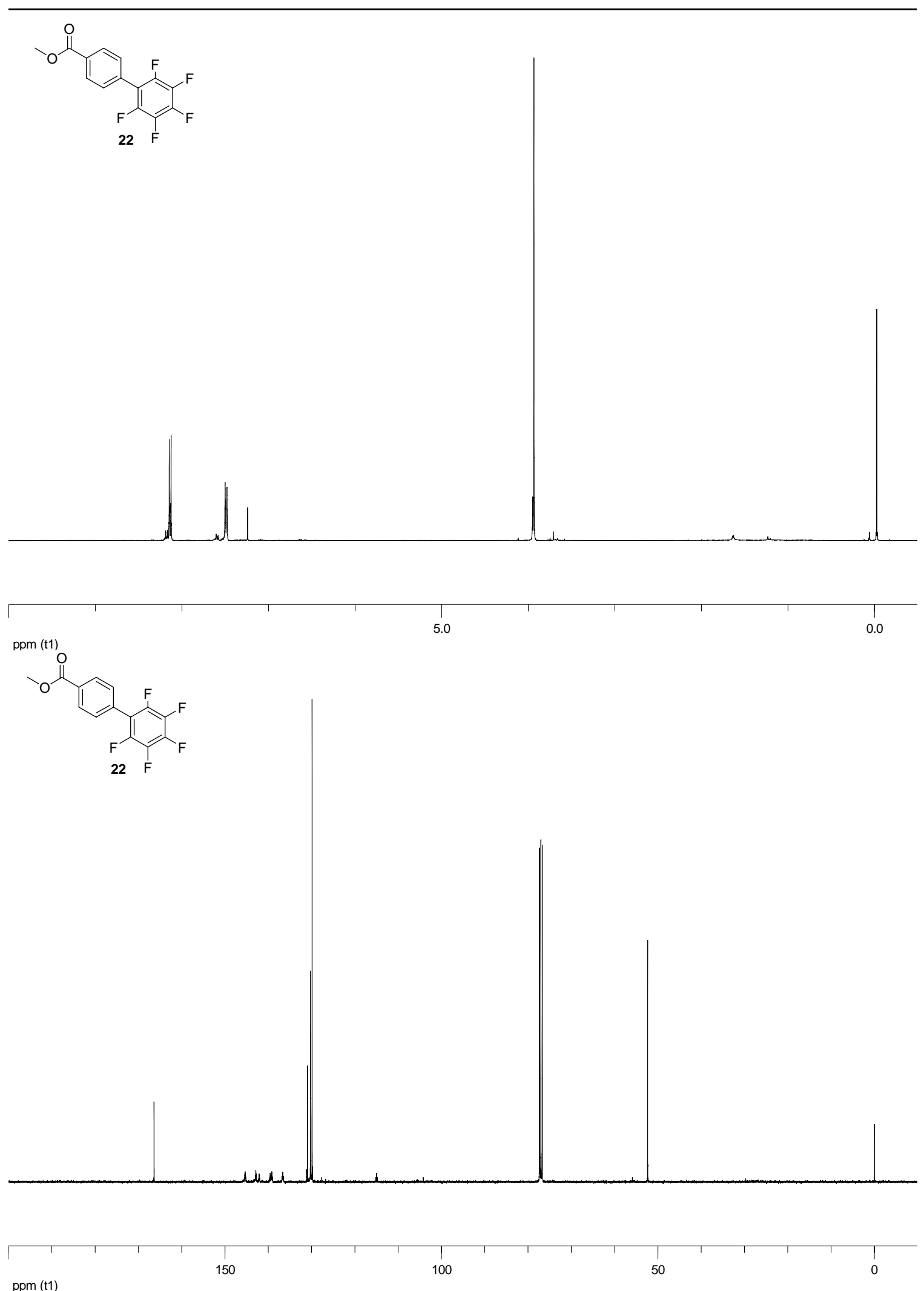

ppm (t1) 


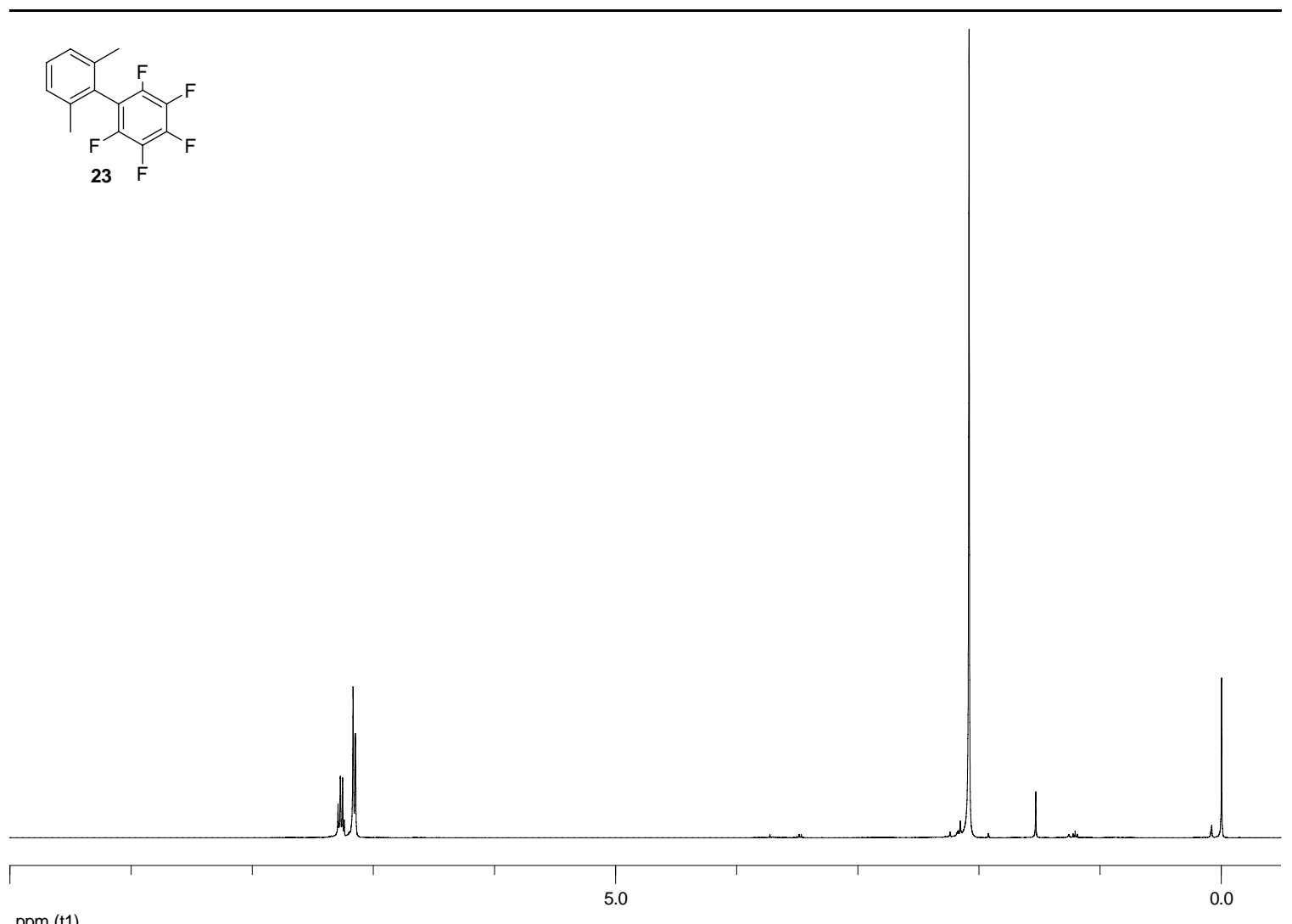

ppm (t1)
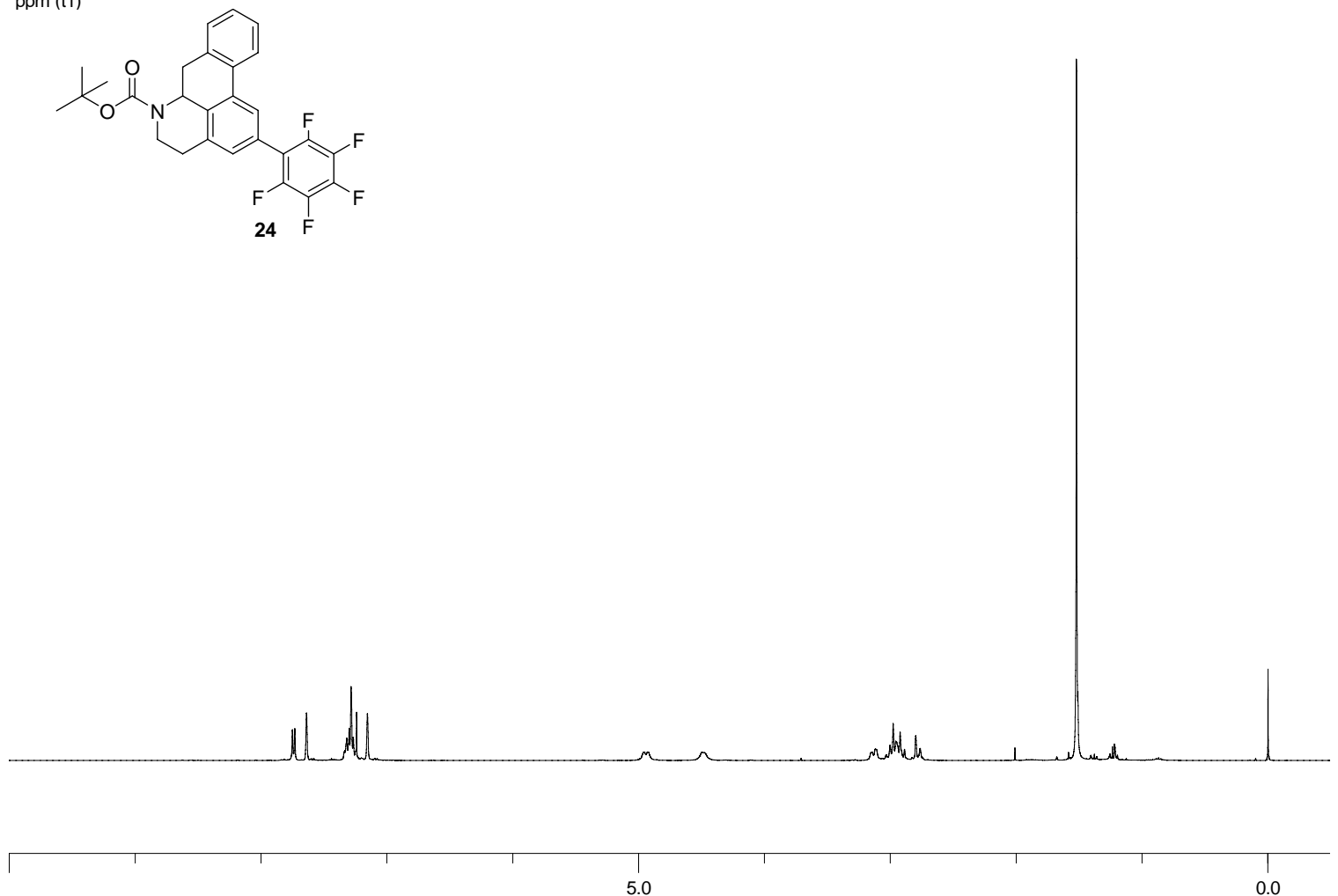

ppm (t1) 

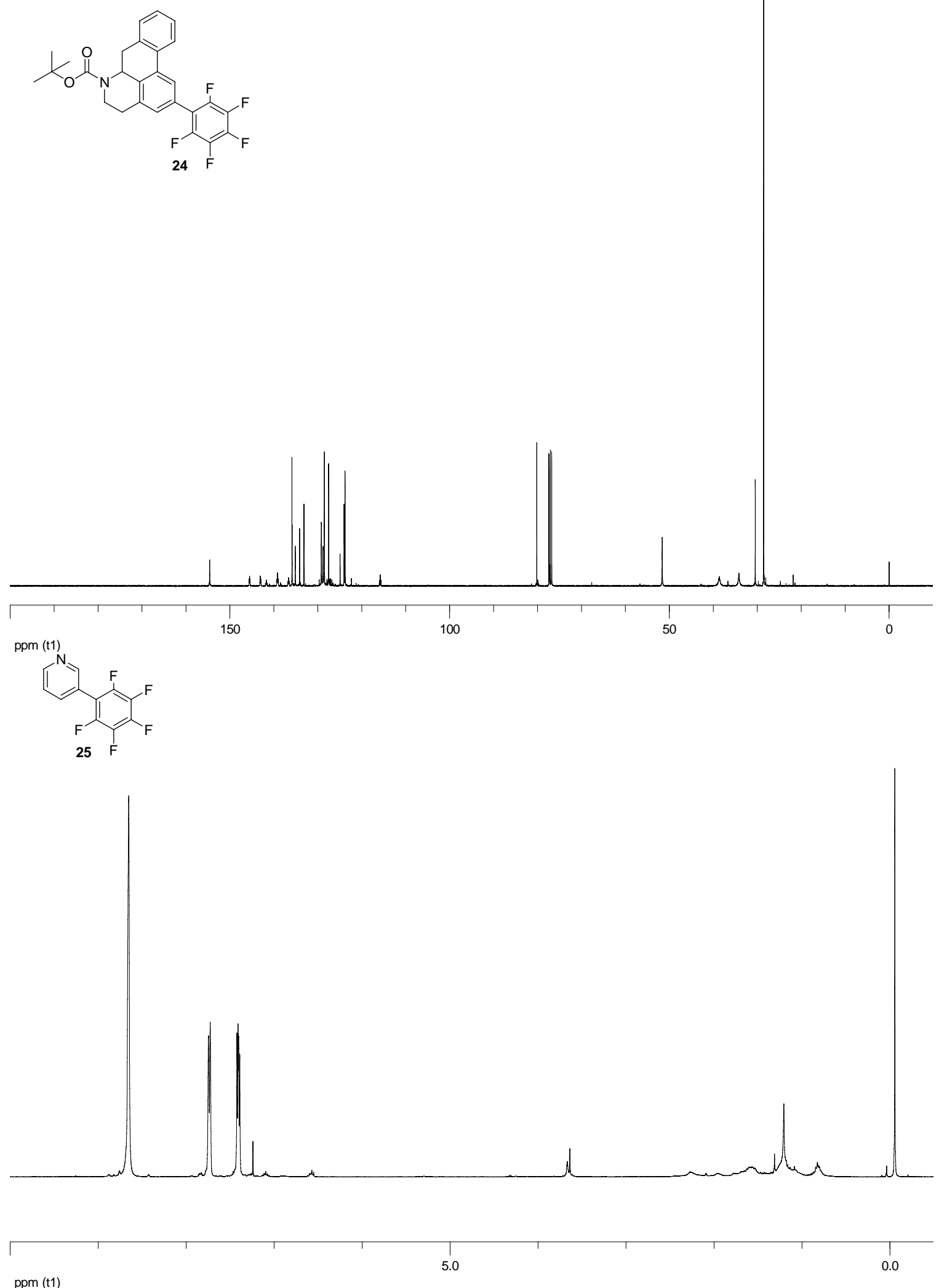

ppm (t1) 

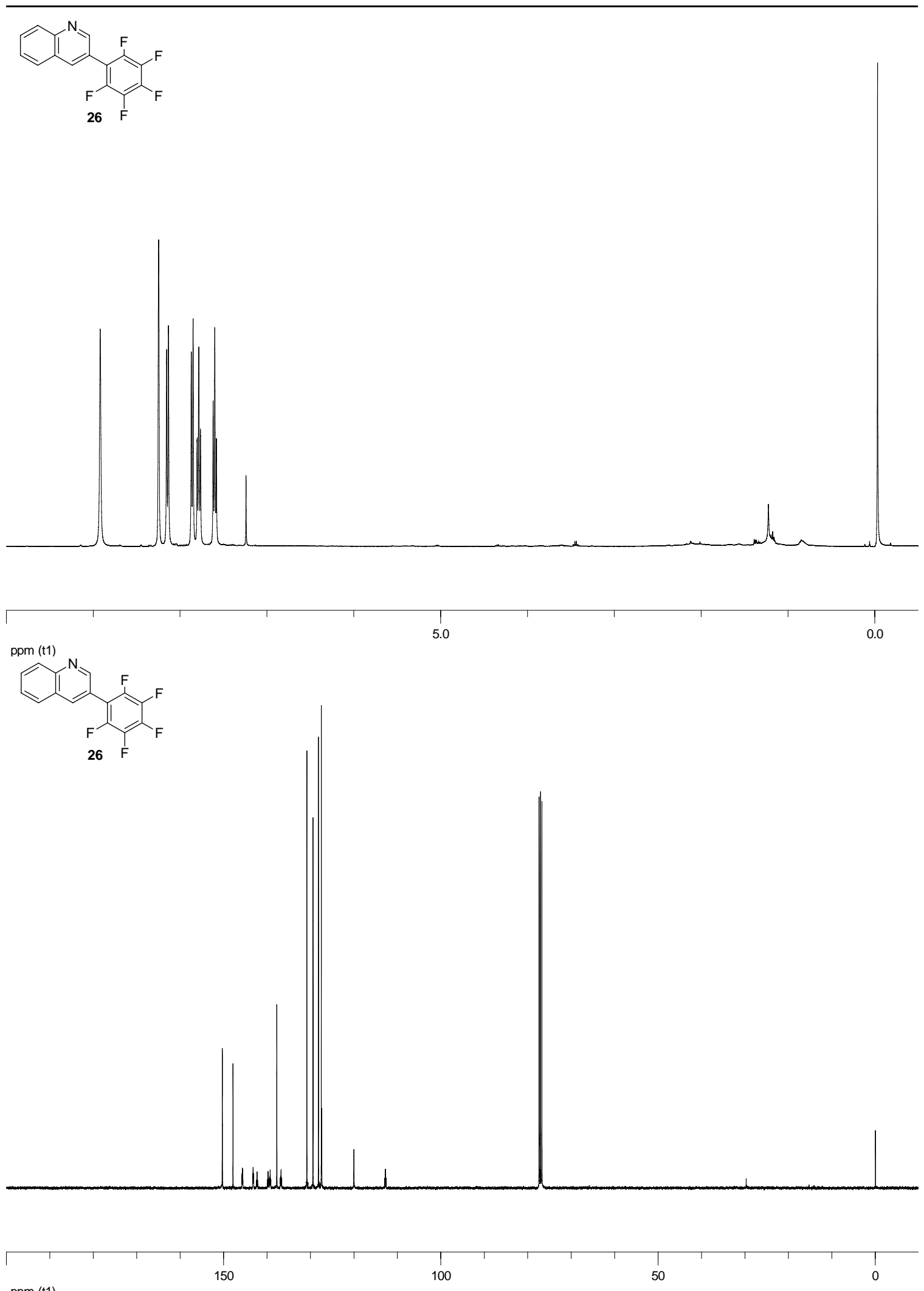

ppm (t1) 

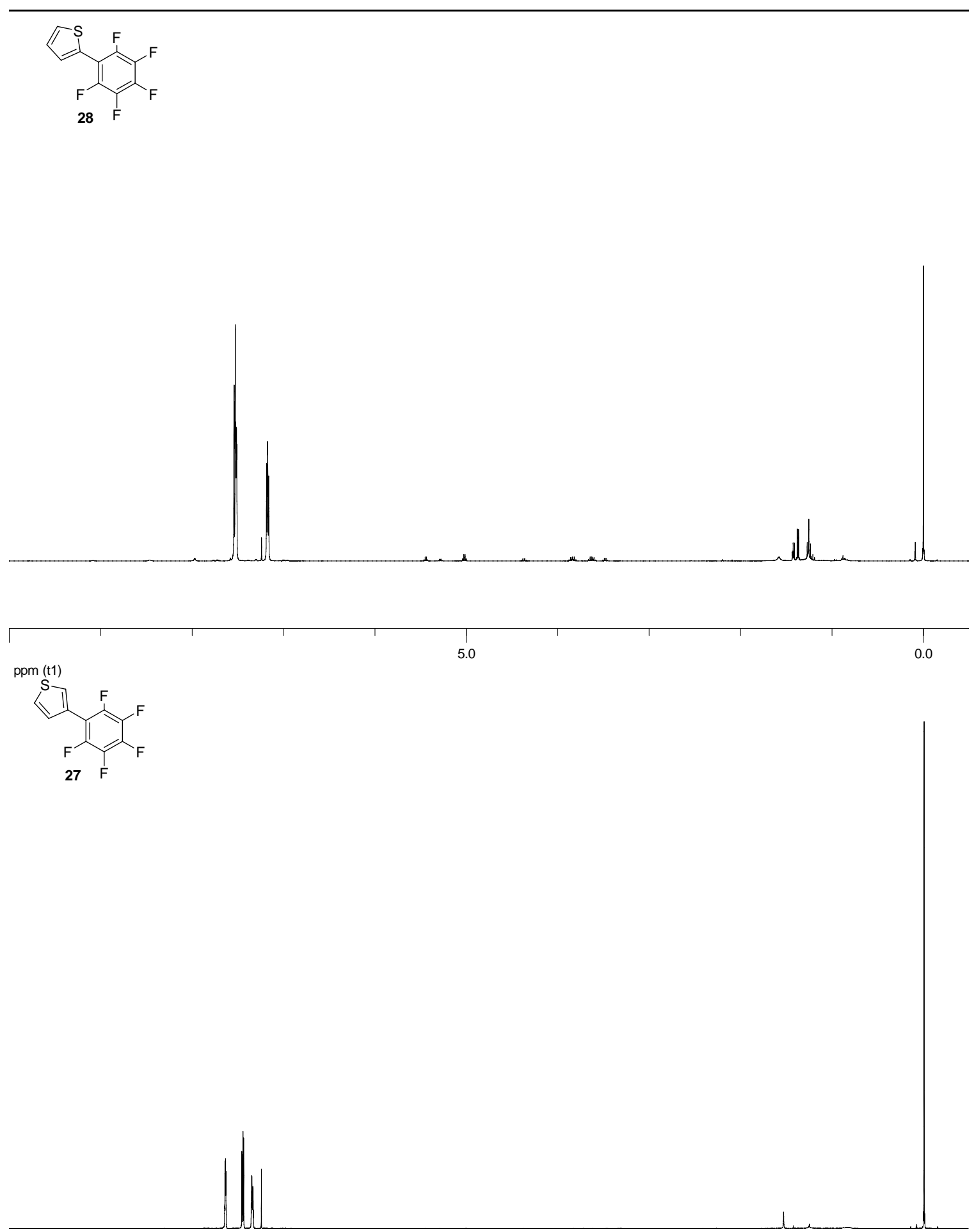

ppm (t1)
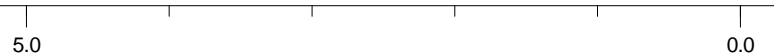
S32
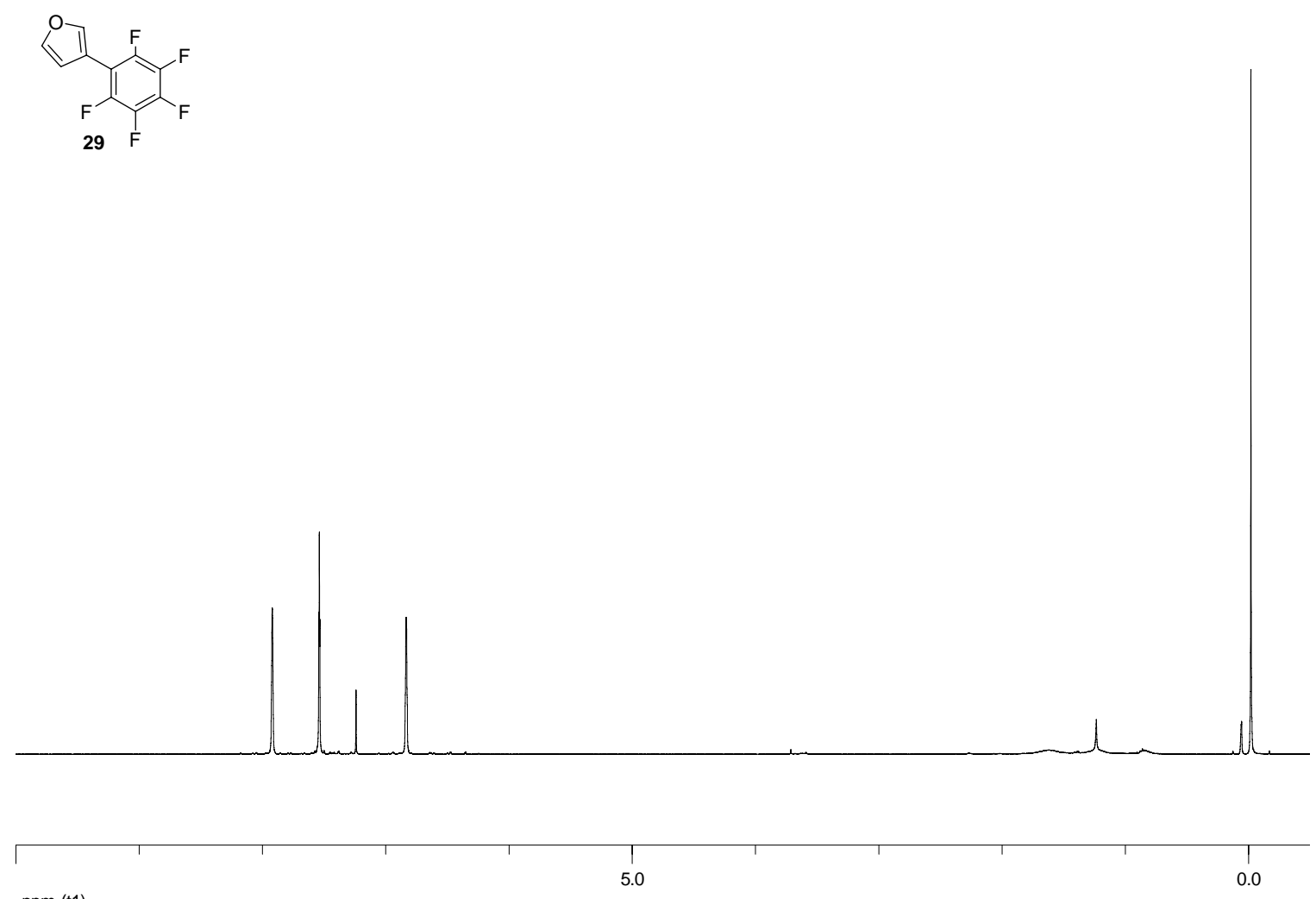

ppm (t1)
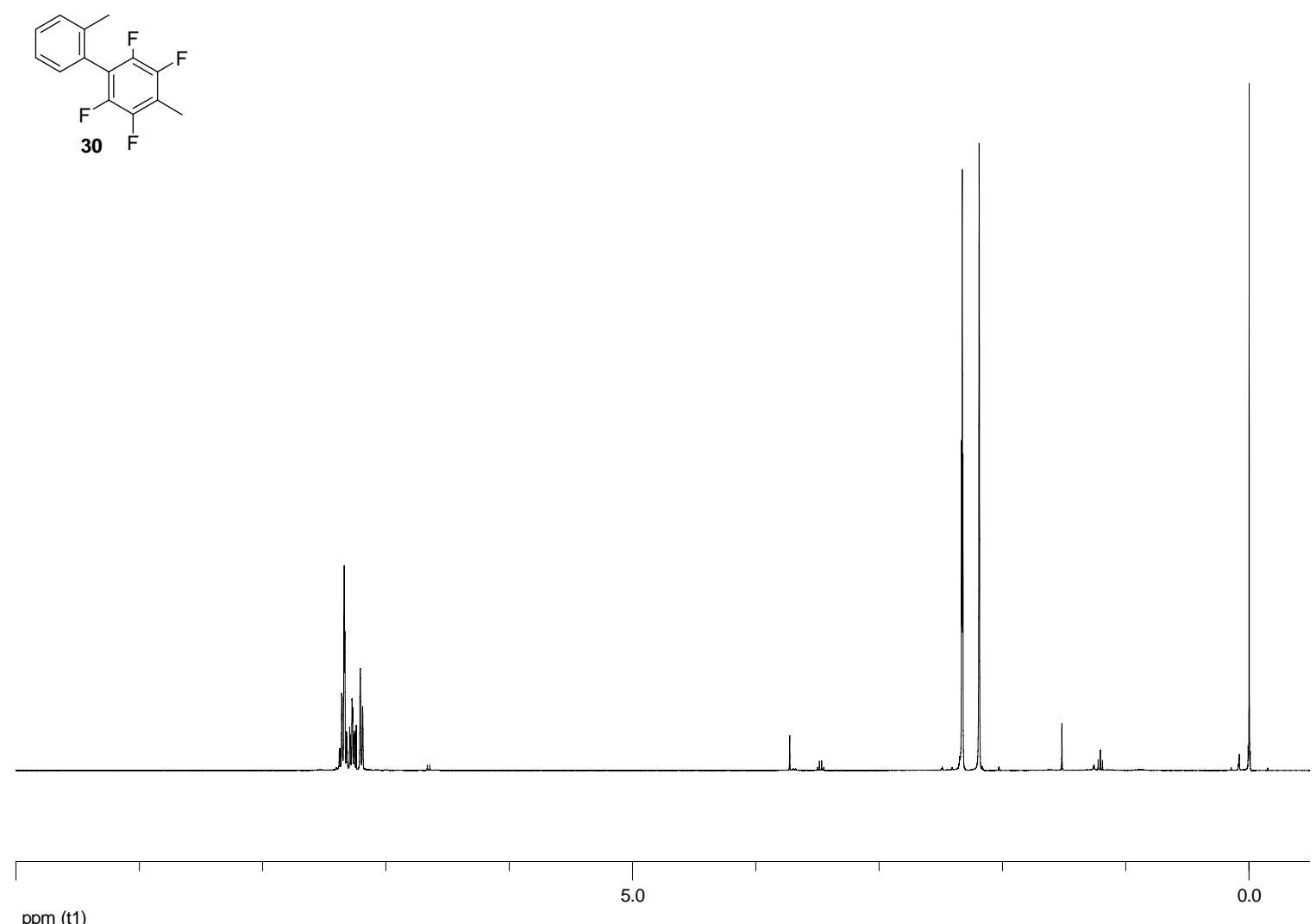

ppm (t1) 
S33
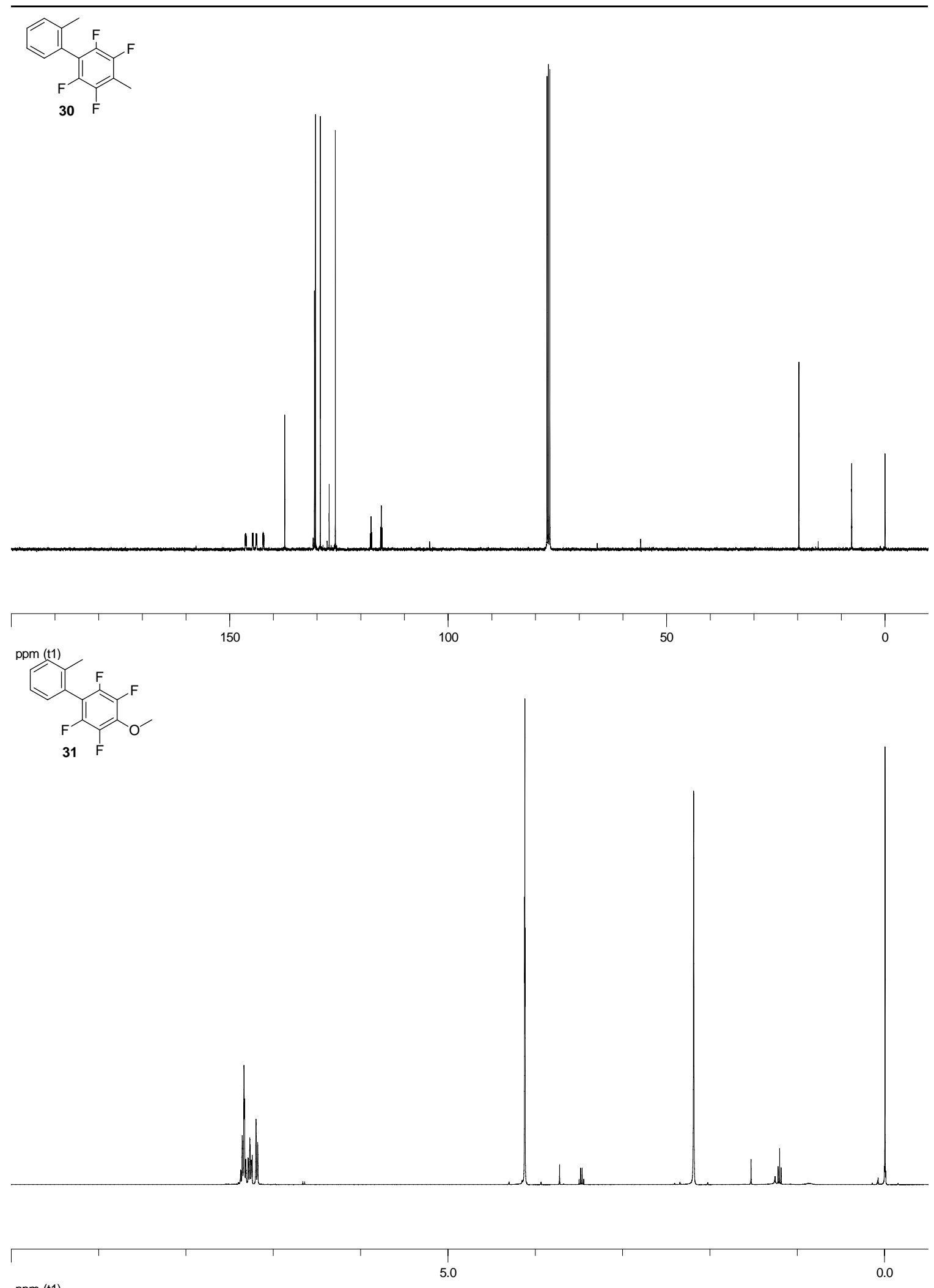
S34
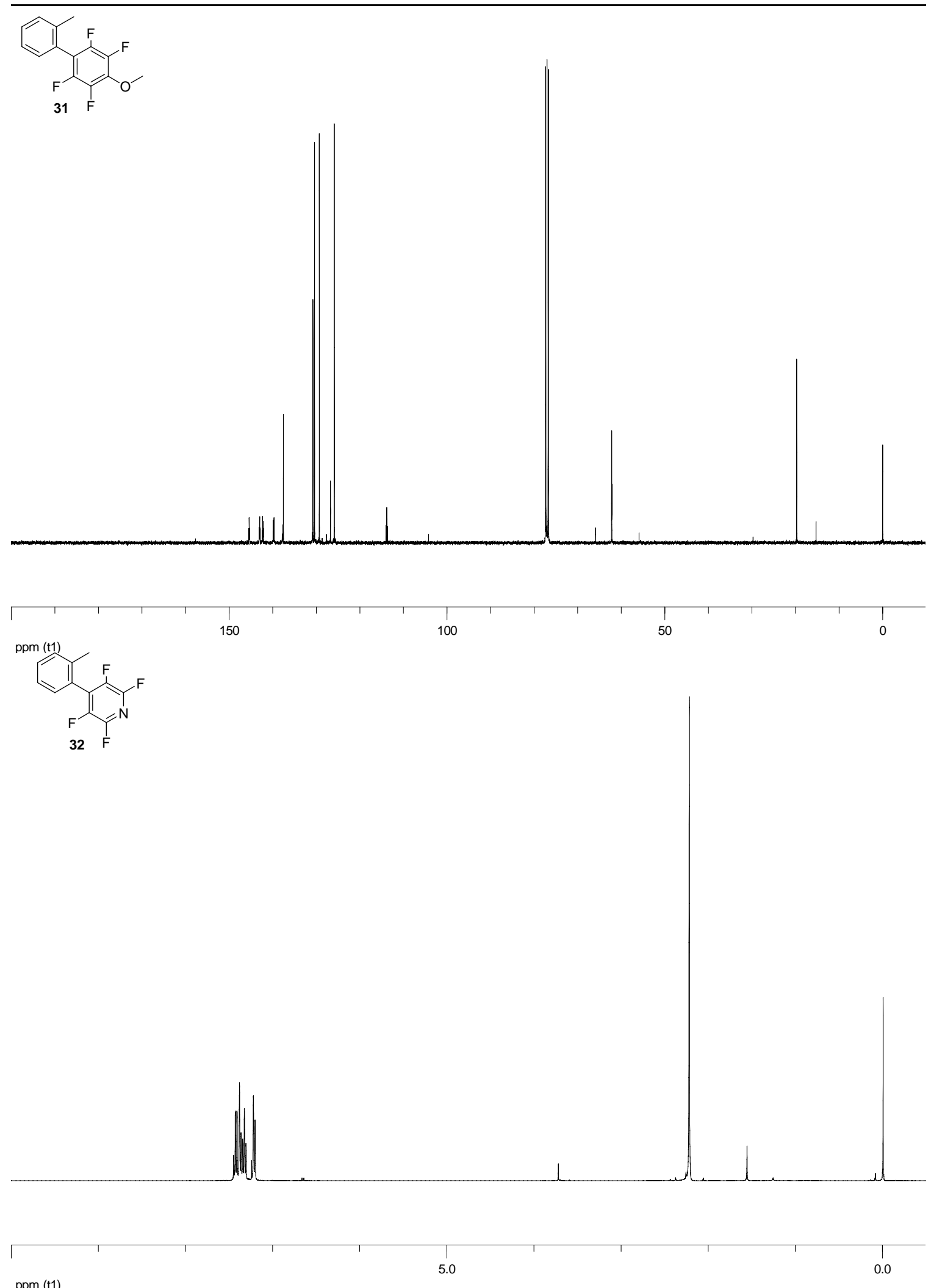

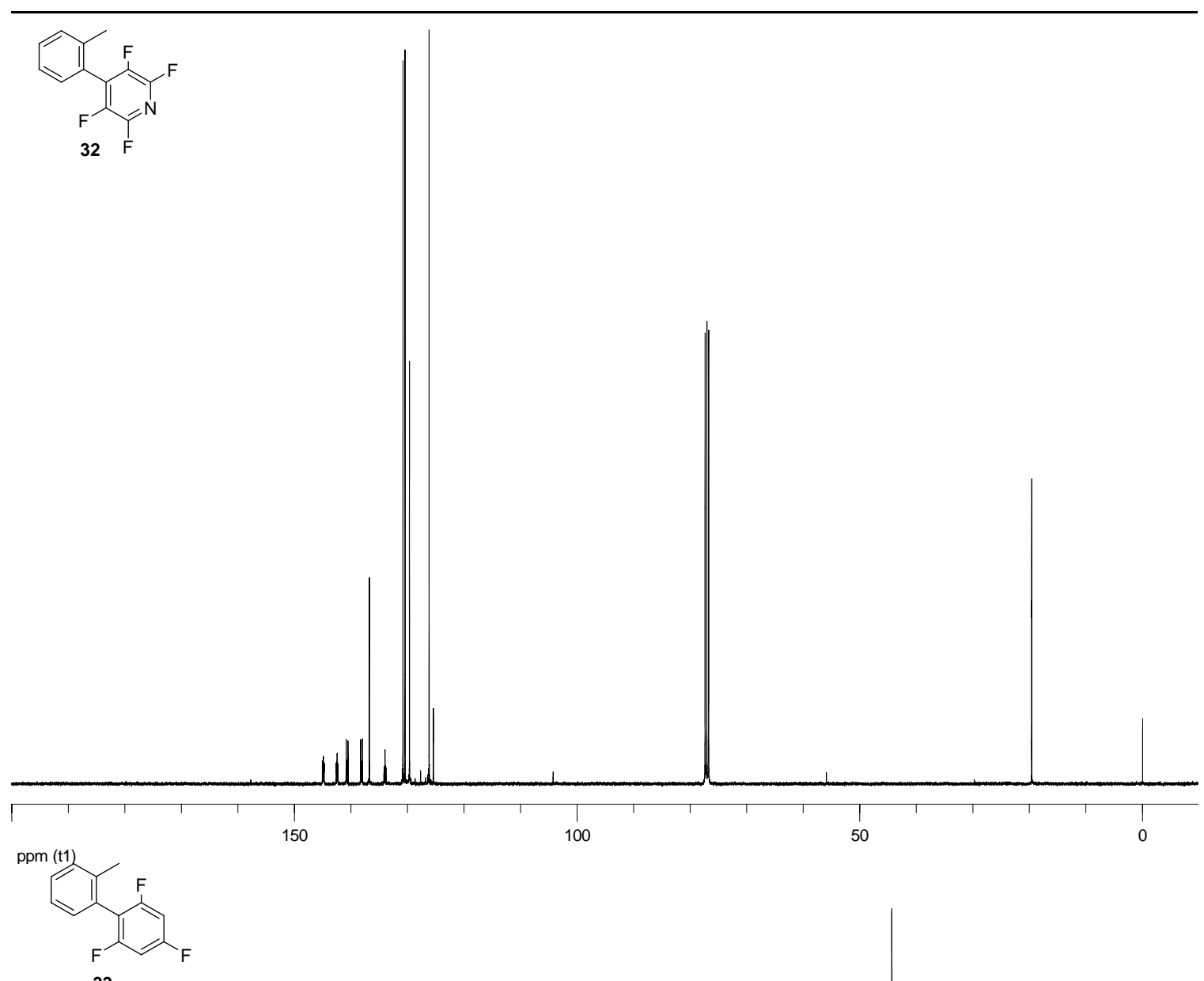

33

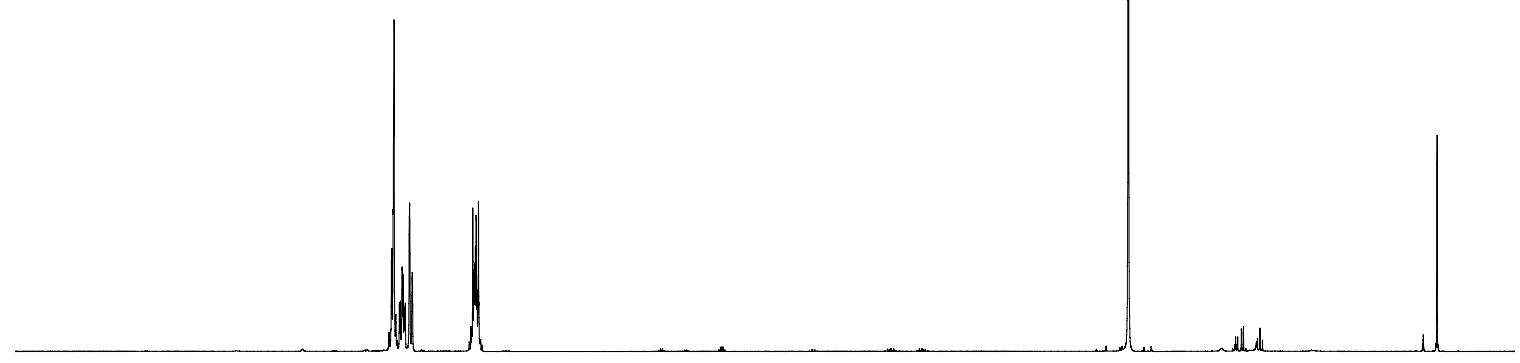

ppm (t1) 


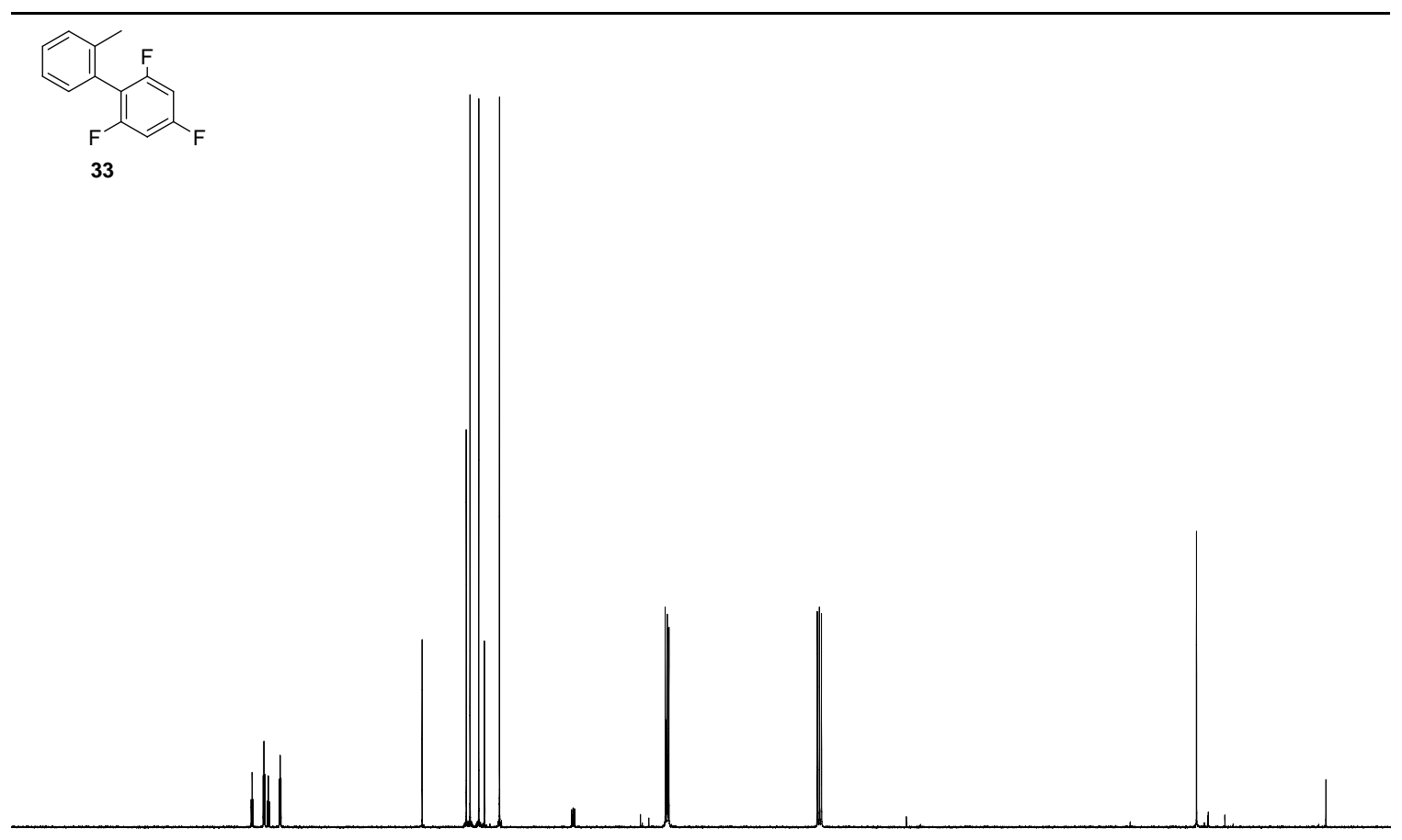

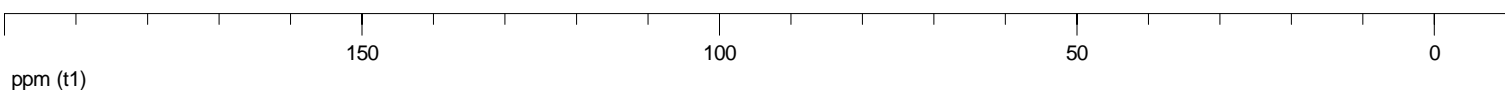

